\title{
Wilson's map operations on regular dessins and cyclotomic fields of definition
}

\author{
G. A. Jones, M. Streit and J. Wolfart
}

\begin{abstract}
Dessins d'enfants can be seen as bipartite graphs embedded in compact orientable surfaces. According to Grothendieck and others, a dessin uniquely determines a complex structure on the surface, even an algebraic structure as a projective algebraic curve defined over a number field. Combinatorial properties of the dessin should therefore determine the equations and also structural properties of the curve, such as the field of moduli or the field of definition. However, apart from a few series of examples, very few general results concerning such correspondences are known. As a step in this direction, we present a graph theoretic characterisation of certain quasiplatonic curves defined over cyclotomic fields, based on Wilson's operations on maps: these leave invariant the graph but change the cyclic ordering of edges around the vertices, therefore they change the embeddings, and hence the dessins, and hence the conformal and algebraic structure of the underlying curves. Under suitable assumptions, satisfied by many series of regular dessins, these changes coincide with the effect of Galois conjugation. This coincidence allows one to draw conclusions about Galois orbits and fields of definition of dessins. The possibilities afforded by these techniques, and their limitations, are illustrated by a new look at some known examples and a study of dessins based on the regular embeddings of complete graphs.
\end{abstract}

\section{The mathematical framework}

For the last twenty years embeddings of graphs into surfaces have become important for the theory of algebraic curves as an essential tool in the theory of dessins d'enfants. In this first section we briefly recall the necessary definitions and give an outline of the problems and results presented in this paper.

One may introduce dessins d'enfants as hypermaps on compact oriented 2-manifolds. These objects, first studied by Cori [5], have several equivalent algebraic or topological definitions: topologically they are a generalisation of maps, whose underlying graphs are replaced with hypergraphs so that the hyperedges are now allowed to be incident with any finite number of hypervertices and hyperfaces. One way to visualise this concept is to represent a hypermap $\mathcal{D}$ by its Walsh map $W(\mathcal{D})$, a connected bipartite graph $\mathcal{B}$ embedded in a compact oriented surface, dividing it into simply connected cells $[\mathbf{2 6}]$; in the language of hypermaps, the white and black vertices represent the hypervertices and hyperedges of $\mathcal{D}$, the edges represent incidences between them, and the cells represent the hyperfaces.

Algebraic hypermaps are an alternative and equivalent way to describe dessins. These are triples $(G, x, y)$ consisting of a permutation group $G \subset S_{n}$ (the monodromy or hypercartographic group of the dessin), acting transitively on the $n$ edges of the Walsh map $W(\mathcal{D})$ and generated by two elements $x$ and $y$. The generators $x$ and $y$ describe the anticlockwise cyclic permutations of the edges around their incident white and black vertices. We call $\mathcal{D}$ a dessin

Received 22 September 2008; revised 9 June 2009.

2000 Mathematics Subject Classification 14H45 (primary), 14H25, 14H55, 05C10, 05C25, 30F10 (secondary).

During the preparation of the article the third author was supported by the Deutsche Forschungsgemeinschaft with the projects Wo $199 / 3$ and Wo $199 / 4$. 
of type $(p, q, r)$ if

$$
p=\operatorname{ord} x, \quad q=\operatorname{ord} y, \quad \text { ord } x y=r .
$$

Geometrically, $p$ and $q$ are the least common multiples of the valencies of the white and black vertices of $\mathcal{D}$; the faces all have even valencies, and their $1 \mathrm{~cm}$ is $2 r$. We call $\mathcal{D}$ a map of type $(p, r)$ in the special case where all black vertices have valency $q=2$. Then we can omit the black vertices, and transform $\mathcal{B}$ into an ordinary graph $\mathcal{G}$ : the vertices of $\mathcal{G}$ are the white vertices of $45 \mathcal{B}$, and its edges are formed from the pairs of edges of $\mathcal{B}$ incident with a black vertex. Now $y$ is a permutation reversing the direction of each dart (directed edge) of $\mathcal{G}$. In this case we call $G$ the cartographic group of the algebraic map $(G, x, y)$. From every hypermap $\mathcal{D}$ we can pass to a map $\mathcal{M}_{\mathcal{D}}$ by forgetting vertex colours in the Walsh representation $W(\mathcal{D})$ and introducing edge directions (the cartographic group will then be a subgroup of $S_{2 n}$ ) and conversely we may consider every map as a hypermap, introducing black midpoints of valency 2 on every edge and replacing the two directions with the two new parts of the edge.

Dessins are linked to Riemann surfaces and algebraic curves in the following way. Riemann surfaces $X$ uniformised by subgroups $\Gamma$ of finite index in triangle groups $\Delta$ play a special role

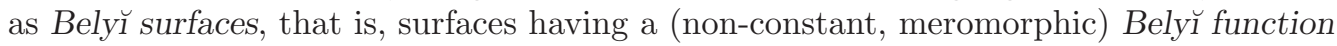

$$
\beta: X \longrightarrow \mathbf{P}^{1}(\mathbf{C})
$$

ramified over at most three points in the Riemann sphere $\mathbf{P}^{1}(\mathbf{C})$, corresponding to the covering map

$$
\beta: \Gamma \backslash \mathbf{H} \longrightarrow \Delta \backslash \mathbf{H}
$$

where $\mathbf{H}$ is the hyperbolic plane if $\Delta$ is a Fuchsian triangle group. In the few cases where

$\Delta$ is a spherical or euclidean triangle group, $\mathbf{H}$ has to be replaced with the Riemann sphere $\mathbf{P}^{1}(\mathbf{C})$ or the Gauss plane $\mathbf{C}$. As first observed by Bely $\breve{1}[\mathbf{2}]$, the existence of such a function is equivalent to the property that $X$, as a smooth projective algebraic curve, can be defined over a number field. Starting with Grothendieck's theory of dessins d'enfants [9], many interesting reformulations of Bely1̌'s theorem have been found; see, for instance, $[\mathbf{1}, \mathbf{1 4}, \mathbf{2 5}]$, the recent survey in $[\mathbf{2 9}]$ or the introduction in $[\mathbf{1 6}]$.

Every Belyı function $\beta$ induces a Walsh map $W(\mathcal{D})$, representing the hypermap $\mathcal{D}$, on the Riemann surface $X$ : if we normalise its critical values to be 0,1 and $\infty$, then $V_{0}=\beta^{-1}(0)$ and $V_{1}=\beta^{-1}(1)$ are the sets of white and black vertices, and the connected components of the preimage of the real interval ]0,1 [ are the edges of the graph; the elements of $V_{\infty}=\beta^{-1}(\infty)$ are called the face centres since there is one in each face of the map. Since the edges of the graph represent the sheets of the covering $\beta$, its monodromy group coincides with the monodromy group of the hypermap $\mathcal{D}$. Conversely, every Walsh map on a compact orientable surface arises in this way from a unique holomorphic structure and a unique Belyu function on the surface. The entries of the triple $(p, q, r)$ giving the type of the resulting dessin divide the corresponding 75 entries of the signature $\langle\bar{p}, \bar{q}, \bar{r}\rangle$ of the triangle group mentioned above; for simplicity one may take $\Delta=\langle p, q, r\rangle$, and we will always assume $\Delta$ to be given in this way. In the special case where all zeros of $1-\beta$ are of order 2 (a clean Belyu function) we get a hypermap of type $(p, 2, r)$, and hence a map of type $(p, r)$. In the language of Belyı functions the passage explained above from Walsh maps to maps is induced by replacing $\beta$ with $4 \beta(1-\beta)$; this is in fact a new and so moreover a clean Belyı function whose zero set is the full vertex set $V_{0} \cup V_{1}$ of the original $\operatorname{dessin} \mathcal{D}$.

A fundamental problem is that of relating the combinatorial properties of the hypermap $\mathcal{D}$ to algebraic properties of the curve $X$, such as its moduli field, Galois orbit, defining equations, etc. In general, this problem is very difficult, but it is a little easier if the dessin has a large

85 automorphism group; for the definition of this in terms of the monodromy group, see the proof of Lemma 3 below. More precisely, we assume that the Belyu function is a regular covering, 
that is, $\beta$ is the quotient map $X \rightarrow A \backslash X$ by a group $A$ of holomorphic automorphisms of the Riemann surface $X$; this is equivalent to $\Gamma$ being a normal subgroup of the triangle group $\Delta$, with $A \cong \Delta / \Gamma$, and also to the hypermap $\mathcal{D}$ being regular, that is, having an automorphism group, isomorphic to $A$, acting transitively on the edges of the Walsh map $W(\mathcal{D})$. It then follows that $X$ has genus

$$
g=1+\frac{|A|}{2}\left(1-\frac{1}{p}-\frac{1}{q}-\frac{1}{r}\right) .
$$

Such surfaces $X$, known as quasiplatonic surfaces, have many interesting properties; see, for instance, [29, Theorem 4]. In particular $A$ can be identified with the Galois group of the extension of function fields corresponding to $\beta$, and the Galois correspondence allows information about $A$ and its action on $X$ to be translated into information about this extension (see, for example, $[\mathbf{6}, \mathbf{1 6}, \mathbf{2 1}-\mathbf{2 3}]$ ). All known examples follow a common pattern, and in the present paper, we will work out the mathematical structure behind it: a correspondence between Galois actions on cyclotomic fields on the one hand and Wilson's map operations $H_{j}$ on the other hand. These act on maps by raising the generating monodromy rotation of edges around each vertex to its $j$ th power, where $j$ is coprime to the valencies of the vertices; we generalise this to an operation $H_{i, j}$ that acts on hypermaps by raising the permutations $x$ and $y$ around the white and black vertices to their $i$ th and $j$ th powers. After explaining these operations in the next section, we will establish this correspondence in Section 3, Theorems 1-3. With slightly simplified hypotheses and statements, their content can be summarised in the following main theorem.

Main Theorem. A Galois invariant family $\left\{\mathcal{D}_{j}\right\}$ of regular dessins forming an orbit under Wilson's operations is defined over a cyclotomic field, and the Wilson operations are equivalent to the Galois conjugations.

Conversely, let $\mathcal{D}_{j}$ run over a family of Galois conjugate regular dessins defined over a cyclotomic field, and suppose that the Galois conjugations preserve adjacency between the vertices of the dessins. Then the algebraic conjugations act as Wilson's operations on the dessins.

We apply these results to examples in Sections 4 and 5. Section 4 sheds new light on some series of known Galois orbits of regular dessins, and in Section 5, we consider regular maps and hypermaps whose underlying graphs are complete graphs $K_{n}$ (for their regular embeddings, see $[\mathbf{3}, \mathbf{1 1}, \mathbf{1 2}])$. In all these examples, Galois conjugation in some sense imitates the effect of Wilson's operations. However, this is not a universal phenomenon, and the Appendix contains a lemma about automorphism groups of graphs and maps, which allows us to show that Galois conjugation and Wilson's operations act very differently on the family of Macbeath-Hurwitz curves. At the end of Section 4, Proposition 3 gives us an indication of how to treat those cases for which the correspondence between Wilson's operations and Galois conjugations no longer applies.

\section{Wilson's operations}

Wilson's operations $H_{j}$ (see $[\mathbf{2 7}]$ ) can be defined as follows. Let $\mathcal{M}$ be a compact oriented map of type $(p, r)$ and let $j$ be an integer; we restrict our considerations to the case where $j$ is coprime to $p$. Each face of $\mathcal{M}$ is bounded by a closed path that, at each vertex, proceeds from one edge to the next incident edge in the anticlockwise direction, so that the path goes clockwise around the face. Thus $\mathcal{M}$ is uniquely determined by its underlying graph $\mathcal{G}$ and by the anticlockwise cyclic rotation $\rho$ of edges around each vertex. Instead, consider paths in $\mathcal{G}$ that, at each vertex, proceed from one edge to the $j$ th incident edge in the anticlockwise direction. Of course these paths are all closed, and they form the boundaries of the faces of a 
new map $H_{j} \mathcal{M}$ that is also compact and oriented: it has the same underlying graph $\mathcal{G}$ as $\mathcal{M}$ but $\rho$ has been replaced with $\rho^{j}$. Each such operation depends only on the congruence class of $j$ modulo $p$; since $H_{j} \circ H_{k}=H_{j k}$ for all $j$ and $k$ coprime to $p$, these operations represent an action of the multiplicative group $(\mathbf{Z} / p \mathbf{Z})^{*}$ of units $\bmod p$. In particular, $H_{-1}$ transforms a map into its mirror image, by reversing the orientation. For further properties of these operations, see $[\mathbf{1 8}, \mathbf{2 7}]$.

We apply this procedure to dessins $\mathcal{D}$ via the maps $\mathcal{M}_{\mathcal{D}}$ introduced above, forgetting the colours of the vertices. The generators $x$ and $y$ of the monodromy group $G$ of $\mathcal{D}$ represent the rotations of edges around the white and black vertices of $W(\mathcal{D})$, and hence they must be replaced with $x^{j}$ and $y^{j}$, where $j$ is coprime to $p q$. Thus we have the following lemma (see [18, Section 7]).

Lemma 1. Let $\mathcal{D}$ be a dessin of type $(p, q, r)$, presented as an algebraic hypermap $(G, x, y)$ with a permutation group $G<S_{n}$ acting transitively on the $n$ edges of $\mathcal{D}$, generated by the elements $x$ and $y$ of order $p$ and $q$, respectively, such that $x y$ has order $r$. Let $j$ be an integer coprime to $p q$. Then Wilson's operation $H_{j}$ transforms $\mathcal{D}$ into the dessin $H_{j} \mathcal{D}$ with the algebraic hypermap $\left(G, x^{j}, y^{j}\right)$.

In the case where $q=2$, such that the dessin $\mathcal{D}$ is in fact already a map, there may be some confusion as to whether $H_{j}$ should be applied directly to the map $\mathcal{D}$ as in the first paragraph, raising $x$ but not $y$ to its $j$ th power, or to the map $\mathcal{M}_{\mathcal{D}}$ as in the second paragraph, raising both $x$ and $y$ to their $j$ th powers. In fact there is no real problem: if $j$ is odd, then $y^{j}=y$, and if $j$ is even (so that $p$ is odd), then we can replace $H_{j}$ with $H_{j+p}$, again fixing $y$.

Note that in Lemma 1, although $x^{j}$ and $y^{j}$ have order $p$ and $q$, respectively, $x^{j} y^{j}$ need not have order $r$, and hence $H_{j} \mathcal{D}$ will have type $\left(p, q, r^{\prime}\right)$ for some $r^{\prime}$, possibly distinct from $r$. Thus type and hence genus are not always preserved by $H_{j}$. This is illustrated by the following examples (see also Examples 3 and 8).

ExAmple 1. The modular group $\mathrm{PSL}_{2}(\mathbf{Z})$ is generated by its elements $\pm\left(\begin{array}{ll}1 & 1 \\ 0 & 1\end{array}\right)$ and $\pm\left(\begin{array}{rr}0 & 1 \\ -1 & 0\end{array}\right)$, and so for each integer $p \geqslant 2$, its quotient $G=\mathrm{PSL}_{2}(\mathbf{Z} / p \mathbf{Z})$ is generated by their images $x$ and $y$ in $G$. Since $x, y$ and $x y$ have orders $p, 2$ and 3 , this gives a regular algebraic hypermap $\mathcal{D}=(G, x, y)$ of type $(p, 2,3)$, that is, a regular map of type $(p, 3)$. Now $x^{j} y= \pm\left(\begin{array}{cc}j-1 \\ 1 & 0\end{array}\right)$ has trace $\pm j$, and hence by choosing suitable values of $j$, we can change the conjugacy class of this element; in many cases this also changes its order and hence changes the type and genus of the map $H_{j} \mathcal{D}$. For instance, if $p=5$, so that $\mathcal{D}$ is the icosahedron with $G=\mathrm{PSL}_{2}(\mathbf{Z} / 5 \mathbf{Z}) \cong A_{5}$, then $x^{2} y$ has order 5 , and hence $H_{2} \mathcal{D}$ has type $(5,5)$; it is, in fact, the great dodecahedron, a regular map of genus 4 denoted by $\{5,5 / 2\}$ in [7]. Similarly, if $p=8$ with $G=\mathrm{PSL}_{2}(\mathbf{Z} / 8 \mathbf{Z})$ of order 192 , then $x^{3} y$ has order 6 , and so $H_{3}$ converts a regular map of type $(8,3)$ and genus 5 into one of type $(8,6)$ and genus 21 . These last two maps are 4 -sheeted unbranched coverings of a similar pair of maps of type $(8,3)$ and $(8,6)$ : the first is the map $\{3,4+4\}$ of genus 2 , a double covering of the octahedron branched over its vertices, with the automorphism group as follows:

$$
G=\left\langle x, y, z \mid x^{8}=y^{2}=z^{3}=x y z=\left[x^{4}, z\right]=1\right\rangle \cong \mathrm{GL}_{2}\left(\mathbf{F}_{3}\right)
$$

(see $[\mathbf{1 7}]$ ), while the second, its image under $H_{3}$, has type $(8,6)$ and genus 6 . (The authors are grateful to Steve Wilson for this example.)

ExAmple 2. The symmetric group $S_{n}$ is generated by the $n$-cycle $x=(1,2, \ldots, n)$ and the 175 transposition $y=(1,2)$, with $x y$ an $(n-1)$-cycle, and thus $S_{n}$ is the monodromy group of a regular map $\mathcal{M}$ of type $(n, n-1)$. If $j$ is coprime to $n$, then $x^{j} y$ is the product of two cycles of 
lengths $k$ and $n-k$, where $j k \equiv 1 \bmod n$ and $1 \leqslant k \leqslant n$; these lengths are mutually coprime; thus $x^{j} y$ has order $k(n-k)$ and hence $H_{j} \mathcal{M}$ has type $(n, k(n-k))$. Since $k(n-k)=k^{\prime}\left(n-k^{\prime}\right)$ if and only if $k^{\prime}=k$ or $n-k$, we thus obtain maps of $\phi(n) / 2$ different types. This is the maximum number possible for any map $\mathcal{M}$ of valency $n$ since $H_{j} \mathcal{M}$ and $H_{n-j} \mathcal{M}$ always have the same type.

If $n$ is odd, so that $x \in A_{n}$, then the underlying graph of the map $\mathcal{M}$ is bipartite, with white and black vertices corresponding to the cosets of $\langle x\rangle$ consisting of even and odd permutations, respectively. In this case $\mathcal{M}$ is the Walsh map of a regular hypermap $\mathcal{D}=\left(A_{n}, x, x^{y}\right)$ of type $(n, n,(n-1) / 2)$ such that $H_{j} \mathcal{D}$ has type $(n, n, k(n-k) / 2)$, and thus we again obtain $\phi(n) / 2$ different types.

In view of examples like this, and to determine whether the hypotheses of Theorem 2 in the next section are satisfied, we need a sufficient condition for $\mathcal{D}$ and $H_{j} \mathcal{D}$ to have the same type. A Frobenius group is a semidirect product $G$ of a normal subgroup $K$ (the Frobenius kernel) by a subgroup $H$ (the Frobenius complement) that acts fixed-point freely by conjugation on $K$, that is such that no pair of non-identity elements of $H$ and $K$ commute with each other. Equivalently, $G$ is a transitive permutation group in which only the identity element fixes more than one point: here $H$ is the stabiliser of a point and $K$ consists of the identity and the elements without fixed points (see $[\mathbf{1 0}, \S \mathrm{V} .8]$ for details). A useful example is the group $\operatorname{AGL}_{1}\left(\mathbf{F}_{n}\right)$ of affine transformations $t \mapsto a t+b\left(a, b \in \mathbf{F}_{n}\right.$, with $\left.a \neq 0\right)$ of a finite field $\mathbf{F}_{n}$ of order $n$, with $K$ (isomorphic to the additive group of $\mathbf{F}_{n}$ ) consisting of the translations $t \mapsto t+b$ and $H$ (the stabiliser of 0 , isomorphic to the multiplicative group $\mathbf{F}_{n}^{*}$ ) consisting of the transformations $t \mapsto a t$, with $a \neq 0$.

Lemma 2. Let $G$ be a finite Frobenius group with a kernel $K$ and a complement $H$. Then two elements of $G \backslash K$ are conjugate in $G$ if and only if their images in $H$ are conjugate in $H$. In particular, all elements of a given coset $K g \neq K$ of $K$ in $G$ have the same order.

Proof. Each element $g \in G$ has the unique form $k h$, where $k \in K$ and $h \in H$, so that $h$ is the image of $g$ under the natural epimorphism $G \rightarrow G / K \cong H$. By applying this epimorphism we see that conjugate elements of $G$ have conjugate images in $H$, and thus each conjugacy class of $G$ is contained in a set $K C$, where $C$ is a conjugacy class of $H$. Since $G$ is a Frobenius group, we have $C_{G}(h)=C_{H}(h)$ for each non-identity $h \in H$, so if $C$ is the conjugacy class of $h$ in $H$, the number of conjugates of $h$ in $G$ is

$$
\left|G: C_{G}(h)\right|=\left|G: C_{H}(h)\right|=|G: H| \cdot\left|H: C_{H}(h)\right|=|K| \cdot|C|=|K C| .
$$

Thus $K C$ is a single conjugacy class of $G$, and hence the first assertion is proved. The second assertion follows immediately.

Corollary 1. Let $\mathcal{D}=(G, x, y)$ be a regular hypermap of type $(p, q, r)$ for which the monodromy group $G$ is a Frobenius group with an abelian complement $H$ and a kernel $K$ not containing $x y$. If $j$ is coprime to $p q r$, then $H_{j} \mathcal{D}$ also has type $(p, q, r)$.

Proof. Since $j$ is coprime to both $p$ and $q$ it is sufficient to show that $x^{j} y^{j}$ has the same order as $x y$, namely $r$. Since $G / K$ is abelian, $x^{j} y^{j}$ is in the same coset of $K$ as $(x y)^{j}$. Now $j$ is coprime to $r$, and hence $(x y)^{j}$ has order $r$ and $(x y)^{j} \notin K$ since $x y \notin K$. The second part of Lemma 2 therefore implies that $x^{j} y^{j}$ has order $r$.

The following example shows that this result does not apply to all Frobenius groups. 
ExAmPle 3. For each prime $p \equiv \pm 1 \bmod 5$, the group $H:=\mathrm{SL}_{2}\left(\mathbf{F}_{5}\right)$ is isomorphic to a subgroup of $\mathrm{SL}_{2}\left(\mathbf{F}_{p}\right)$. The resulting action of $H$ on $K:=C_{p} \times C_{p}$ (regarded as a twodimensional vector space over $\mathbf{F}_{p}$ ) determines a semidirect product $G$ of $K$ by $H$, and this is a Frobenius group with a non-abelian complement $H$ (see $[\mathbf{1 0}$, V.8.8(b)]). The generators $a=\left(\begin{array}{ll}1 & 0 \\ 1 & 1\end{array}\right)$ and $b=\left(\begin{array}{ll}1 & 1 \\ 0 & 1\end{array}\right)$ of $H$ have order 5 , with $a b$ and $a^{2} b^{2}$ of order 10 and 6 , respectively. Let $S$ be the subgroup of $G$ generated by $x:=a$ and $y:=k b$ for some non-identity element $k \in K$. Since $S$ maps onto $H$, we have $G=K S$. If $K \cap S=1$, then $S$ is a complement for $K$ in $G$; however, all such complements are conjugate (since $K$ and $H$ have coprime orders; see $[10$, I.18.3]), and are therefore point-stabilisers, whereas $x$ and $y$ each fix a single distinct fixed point, and so $K \cap S>1$. Now $K \cap S$ is a normal subgroup of $S$, and $S$ acts irreducibly on $K$ since $H$ does, so $K \cap S=K$. Thus $S \geqslant K$, therefore $S=K S=G$ and hence $x$ and $y$ generate $G$. By Lemma 2 the resulting hypermap $\mathcal{D}=(G, x, y)$ has type $(5,5,10)$, whereas $H_{2} \mathcal{D}$ has type $(5,5,6)$.

The case where $x y \in K$ is dealt with by the following slightly more general result.

Proposition 1. Let $\mathcal{D}=(G, x, y)$ be a regular hypermap of type $(p, q, r)$ for which $x y$ lies in an abelian normal subgroup $K$ of $G$. If $j$ is coprime to $p q r$, then $H_{j} \mathcal{D}$ also has type $(p, q, r)$.

Proof. As in the proof of Corollary 1 it is sufficient to show that the order $r^{\prime}$ of $x^{j} y^{j}$ is equal to the order $r$ of the element $w=x y$. Now

$$
x^{j} y^{j}=x^{j} \cdot w^{x} \cdot w^{x^{2}} \cdot \ldots \cdot w^{x^{j}} \cdot x^{-j},
$$

which is conjugate (by $x^{j-1}$ ) to the element $v=w \cdot w^{x} \cdot w^{x^{2}} \cdot \ldots \cdot w^{x^{j-1}}$, and thus $v$ has order $r^{\prime}$. Since $w$ and all its conjugates lie in $K$ they commute with each other; thus $v^{r}=1$ and hence $r^{\prime}$ divides $r$. If $r^{\prime}<r$, then let $L$ be the characteristic subgroup of $K$ consisting of its elements of order dividing $r^{\prime}$, and hence $w, w^{x}, \ldots, w^{x^{j-1}} \in K \backslash L$, whereas $v \in L$. Now $w^{x} \cdot w^{x^{2}} \cdot \ldots$. $w^{x^{j-1}} \cdot w^{x^{j}}=v^{x} \in L$; thus $w^{x^{j}} \equiv w \bmod L$ and hence the elements $w, w^{x}, \ldots, w^{x^{j-1}}$ represent an orbit of length $l$ dividing $j$ in the action of $\langle x\rangle$ by conjugation on $K / L$. Since $j$ is coprime to the order $p$ of $x$, it follows that $l=1$, so $w \equiv w^{x} \equiv \ldots \equiv w^{x^{j-1}} \bmod L$. Thus $w^{j} \equiv v \bmod L$,

245 and hence $w^{j} \in L$; since $j$ is coprime to $r$, it follows that $w \in L$, which is a contradiction. Thus $r^{\prime}=r$, as required.

Sometimes the following more general version of Wilson's operations is useful, as we shall see later in Lemma 4, Theorem 5 and Examples 5, 7 and 10.

Definition 1. Let $\mathcal{D}$ be a dessin of type $(p, q, r)$ and suppose that $i$ is coprime to $p$, and $j$ is coprime to $q$. We define $H_{i, j} \mathcal{D}$ to be the dessin resulting from $\mathcal{D}$ by an application of the $H_{i}$ procedure to the white vertices and of $H_{j}$ to the black vertices. In other words, the algebraic hypermap $(G, x, y)$ is replaced with $\left(G, x^{i}, y^{j}\right)$.

(As in Wilson's original definition, one could even define $H_{i, j}$ for arbitrary integers $i$ and $j$.) The above definition induces an action of $(\mathbf{Z} / p \mathbf{Z})^{*} \times(\mathbf{Z} / q \mathbf{Z})^{*}$ on dessins with the same monodromy group and embedded graph, commuting with the action of the usual Wilson operations defined earlier. Namely, if $k$ is coprime to $p q$, then we have $H_{k} H_{i, j}=H_{i, j} H_{k}=$ $H_{k i, k j}$ and $H_{k}=H_{k, k}$. Note that $H_{1 .-1}$ and $H_{-1,1}$ act as Petrie operations, transposing faces and Petrie polygons (closed zig-zag paths); they differ by a reversal of the orientation. 


\section{The main results}

The next two Lemmas are certainly known to experts, but since they do not seem to be 260 formulated explicitly in the literature, we state them here and give a short proof.

LEMma 3. Let $\mathcal{D}$ be a regular dessin of type $(p, q, r)$, presented as an algebraic hypermap $(G, x, y)$ with a transitive permutation group $G<S_{n}$ acting on the $n$ edges of $\mathcal{D}$, generated by the elements $x$ and $y$ of order $p$ and $q$, respectively. Its monodromy group $G$ and its automorphism group $A \leqslant S_{n}$ determine each other uniquely by

$$
A=C(G) \text { and } \quad G=C(A)
$$

where $C$ denotes the centraliser in $S_{n}$.

Proof. It is well known that, as a permutation group on the set $E(\mathcal{D})$ of edges, $A=C(G)$; see, for example, [14]. This immediately implies that $G \leqslant C(A)$. Since the dessin $\mathcal{D}$ is regular, $A$ acts transitively on $E(\mathcal{D})$. It follows that $C(A)$, as a permutation group with a transitive centraliser, must act without fixed points on the set of edges. It therefore contains at most one element sending any given edge to another. However, its subgroup $G$ always contains such an element, and hence $C(A)=G$.

REMARK 1. A regular dessin $\mathcal{D}$ does not necessarily lead to a regular map $\mathcal{M}_{\mathcal{D}}$ since the

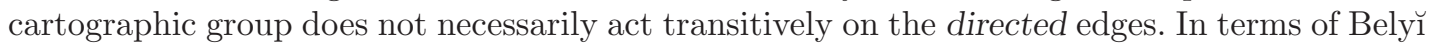
functions, the regularity of $\beta$ as a covering map does not imply the regularity of $4 \beta(1-\beta)$.

REMARK 2. Since $A=C(G)$ without the regularity assumption, and since $G$ is not changed by Wilson's operation $H_{j}$ (see Lemma 1$), H_{j} \mathcal{D}$ has the same automorphism group as $\mathcal{D}$ for any dessin $\mathcal{D}$.

The automorphism group $A$ of a regular dessin $\mathcal{D}$ of type $(p, q, r)$ is generated by two elements $a_{0}$ and $b_{0}$ of order $p$ and $q$, called canonical generators, which fix a white vertex $P_{0}$ and a neighbouring black vertex $Q_{0}$, respectively, each permuting their incident edges by a cyclic shift to the anticlockwise next edge; by the regularity of $\mathcal{D}$, all such pairs $P_{0}$ and $Q_{0}$ are equivalent under $A$, and thus $a_{0}$ and $b_{0}$ are unique up to conjugacy. Their product $a_{0} b_{0}$, which has order $r$, describes a clockwise rotation of the face of $\mathcal{D}$ on the left-hand side of the edge ${ }^{285}$ from $P_{0}$ to $Q_{0}$ (or of all such faces if there are multiple edges).

LEMmA 4. Let $\Delta=\langle p, q, r\rangle=\left\langle\gamma_{0}, \gamma_{1}, \gamma_{\infty} \mid \gamma_{0}^{p}=\gamma_{1}^{q}=\gamma_{\infty}^{r}=1=\gamma_{0} \gamma_{1} \gamma_{\infty}\right\rangle$ be a triangle group in its usual representation and let $\mathcal{D}$ be the regular dessin defined by the Bely function

$$
\beta: \Gamma \backslash \mathbf{H} \longrightarrow \Delta \backslash \mathbf{H}
$$

where $\Gamma$ denotes the kernel of the canonical epimorphism

$$
h: \Delta \longrightarrow A=\text { Aut } \mathcal{D} \cong \Delta / \Gamma, \quad h\left(\gamma_{0}\right)=a_{0}, \quad h\left(\gamma_{1}\right)=b_{0}
$$

Suppose that $i$ and $j$ are coprime to $p q$. Let $a_{0}^{i} b_{0}^{j}$ have order $r^{\prime}$ in $A$, let $\Delta^{\prime}=\left\langle p, q, r^{\prime}\right\rangle=$ $\left\langle\delta_{0}, \delta_{1}, \delta_{\infty} \mid \delta_{0}^{p}=\delta_{1}^{q}=\delta_{\infty}^{r^{\prime}}=1=\delta_{0} \delta_{1} \delta_{\infty}\right\rangle$ and let $h^{\prime}: \Delta^{\prime} \rightarrow A$ be the epimorphism defined by

$$
h^{\prime}\left(\delta_{0}\right):=a_{0}^{i} \quad \text { and } \quad h^{\prime}\left(\delta_{1}\right):=b_{0}^{j}
$$


with kernel $\Gamma^{\prime}$. Then $H_{i, j} \mathcal{D}$ is the dessin corresponding to the Belyı̆ function

$$
\beta^{\prime}: \Gamma^{\prime} \backslash \mathbf{H} \longrightarrow \Delta^{\prime} \backslash \mathbf{H}
$$

(For the few signatures not defining Fuchsian triangle groups, $\mathbf{H}$ has to be replaced with the Riemann sphere or the Gauss plane.)

(To apply Lemma 4 to the original Wilson operation, recall that $H_{j}=H_{j, j}$.)

Proof of Lemma 4. Let $\mathcal{D}^{\prime}$ denote the dessin corresponding to $\beta^{\prime}$. Since $\mathcal{D}$ and $\mathcal{D}^{\prime}$ are both regular, with automorphism group $A$, their edge-sets $E(\mathcal{D})$ and $E\left(\mathcal{D}^{\prime}\right)$ are both permuted regularly by $A$. We can therefore identify $E(\mathcal{D})$ with $E\left(\mathcal{D}^{\prime}\right)$ so that $A$ induces the same permutation group on both edge-sets. By Lemma 2, the monodromy groups of $\mathcal{D}$ and $\mathcal{D}^{\prime}$ coincide, and hence these dessins differ only in the local cyclic ordering of edges around the white and black vertices. The edges of each dessin can be identified with the elements of $A$, so that in $\mathcal{D}$ these cyclic orderings are given by right multiplication by the images $a_{0}$ and $b_{0}$ of $\gamma_{0}$ and $\gamma_{1}$, while in $\mathcal{D}^{\prime}$ they are given by the images $a_{0}^{i}$ and $b_{0}^{j}$ of $\delta_{0}$ and $\delta_{1}$, respectively. It immediately follows as in Lemma 1 that $\mathcal{D}^{\prime} \cong H_{i, j} \mathcal{D}$.

Lemma 5. Let $\mathcal{D}$ be a dessin with an automorphism group $A$ and let $\sigma \in \mathrm{Gal} \overline{\mathbf{Q}} / \mathbf{Q}$. Then the conjugate dessin $\mathcal{D}^{\sigma}$ has an automorphism group $A^{\sigma} \cong A$, and the mapping $P \mapsto P^{\sigma}$ induces isomorphisms between the actions of $A$ on the sets $V_{0}, V_{1}$ and $V_{\infty}$ of white and black vertices and face centres $P$ of $\mathcal{D}$ and the actions of $A^{\sigma}$ on the corresponding sets of points $P^{\sigma}$ of $\mathcal{D}^{\sigma}$.

Proof. The automorphisms of $\mathcal{D}$ are also automorphisms of the corresponding curve $X$, and are thus birational transformations of $X$. Like $X$ they are defined over $\overline{\mathbf{Q}}$, and hence $\sigma$ may be applied to them through their coefficients. They are in fact the automorphisms of $X$ serving as covering transformations of the Belyu function $\beta$ corresponding to $\mathcal{D}$, and hence $\sigma$ induces a bijection between the automorphism groups $A$ and $A^{\sigma}$ of $\mathcal{D}$ and $\mathcal{D}^{\sigma}$, respectively. If $a, b \in A$, then the coefficients of $a b$ are rational functions of those of $a$ and $b$, and hence $(a b)^{\sigma}=a^{\sigma} b^{\sigma}$ and this bijection is an isomorphism.

The white vertices, black vertices and face centres of $\mathcal{D}$ are the points $P \in X$ such that $\beta(P)=0,1$ or $\infty$. Since $\beta$ is defined over $\overline{\mathbf{Q}}$, these are all $\overline{\mathbf{Q}}$-rational points of $X$, and thus they are sent bijectively by $\sigma$ to the white vertices, black vertices and face centres $P^{\sigma}$ of $\mathcal{D}^{\sigma}$. The second assertion now follows from the fact that $a(P)=Q$ if and only if $a^{\sigma}\left(P^{\sigma}\right)=Q^{\sigma}$.

REMARK 3. Although Galois conjugation acts in this natural way on the vertices and face centres of a dessin, it does not do so on the edges. The first difficulty is that edges are defined topologically, not algebraically, as connected sets covering the open unit interval ]0, 1 [, and even if one restricts attention to $\overline{\mathbf{Q}}$-rational points of $X$, a point $P$ on an edge of $\mathcal{D}$ may be sent to a point $P^{\sigma}$ not on an edge of $\mathcal{D}^{\sigma}$ since an algebraic number which is in ]0,1[ may be conjugate to one which is not. Of course, edge-points that cover rational elements of ]0,1[ will be sent to edge-points, but two such points on the same edge may be sent to points on different edges. A second related difficulty is that if $P$ and $Q$ are adjacent vertices of $\mathcal{D}$ then the vertices $P^{\sigma}$ and $Q^{\sigma}$ of $\mathcal{D}^{\sigma}$ need not be adjacent: indeed it is this possibility that allows Galois conjugate dessins to have mutually non-isomorphic embedded graphs (see, for instance, Example 8 and Corollary A.1). 
In order to avoid these difficulties we work under additional hypotheses, for example, that the Galois conjugation $\sigma$ is adjacency preserving on $\mathcal{D}$, which means the existence of a white vertex $P_{0}$ and an adjacent black vertex $Q_{0}$ of $\mathcal{D}$ such that $P_{0}^{\sigma}$ and $Q_{0}^{\sigma}$ are neighbours in the Galois conjugate dessin $\mathcal{D}^{\sigma}$. By Lemma 5 and the transitivity of the action of the automorphism group $A$ on $V_{0}$ and $V_{1}$ in the case of regular dessins we have the following lemma.

Lemma 6. Let $\mathcal{D}$ be a regular dessin with automorphism group $A$ and let $\sigma$ be an adjacency preserving Galois conjugation. Then for all adjacent pairs $P$ and $Q$ of white and black vertices in $\mathcal{D}$, their $\sigma$-images $P^{\sigma}$ and $Q^{\sigma}$ in $\mathcal{D}^{\sigma}$ are also adjacent.

Under the hypothesis that $\sigma$ is adjacency preserving, we can extend its action to the edges of $\mathcal{D}$ in a way that is compatible with the action of $A$.

Lemma 7. Let $\mathcal{C}$ and $\mathcal{C}^{\prime}$ denote the underlying graphs of a regular dessin $\mathcal{D}$ and its Galois conjugate $\mathcal{D}^{\sigma}$. If $\sigma$ preserves adjacency on $\mathcal{D}$, then there is a graph isomorphism $f: \mathcal{C} \rightarrow \mathcal{C}^{\prime}$ such that:

(i) $f$ coincides with $\sigma$ on the vertices;

(ii) $f \circ a=a^{\sigma} \circ f$ for all automorphisms $a \in A$.

Proof. If there is only one edge joining the neighbouring vertices $P_{0}$ and $Q_{0}$ in $\mathcal{D}$, then the same is true for their $\sigma$-images in $\mathcal{D}^{\sigma}$, because the existence of multiple edges can be characterised by the existence of a non-trivial subgroup of $A$ fixing both points, and because this property is $\sigma$-invariant (see the last line of the proof of Lemma 5 , with $P=Q$ first a white and then a black vertex). If we exclude this possibility, then edges correspond bijectively to adjacent pairs of vertices, and thus there is an obvious and canonical choice for $f$. If, on the other hand, there are several edges joining adjacent vertices, then the definition of $f$ is still determined canonically on the vertices by property (i), but no longer on the edge set. In this case take an arbitrary edge $e_{0}$ between $P_{0}$ and $Q_{0}$, say, and define $f\left(e_{0}\right)$ to be an edge between $P_{0}^{\sigma}$ and $Q_{0}^{\sigma}$. Since each other edge in $\mathcal{D}$ can be written as $a\left(e_{0}\right)$ for a unique $a \in A$, property (ii) determines $f$ in a unique way. It is easy to see that incidence is preserved, and hence we have a graph isomorphism.

This graph isomorphism $f$ is in general not a dessin isomorphism since it need not preserve the local ordering of the edges around the vertices. To study its behaviour around the vertices, we consider a canonical pair of generators $a_{0}$ and $b_{0}$, fixing a white vertex $P_{0}$ and a neighbouring black vertex $Q_{0}$. Since $A$ can also be regarded as a group of automorphisms of $X$, the action of $a_{0}$ or $b_{0}$ locally around $P_{0}$ or $Q_{0}$ can be written in suitable local coordinates as a rotation

$$
z \longmapsto \zeta_{p} z \text { around } z\left(P_{0}\right)=0 \text { with } \zeta_{p}:=e^{2 \pi i / p}
$$

or $z \mapsto \zeta_{q} z$ around $z\left(Q_{0}\right)=0$, respectively. More generally, if $P$ is any white vertex of $\mathcal{D}$ fixed by $a \in A$ (the stabiliser in $A$ is always cyclic of order $p$ ), then $a$ acts locally around $P$ as multiplication, the only difference being that the multiplier $\zeta_{p}$ must now be replaced with a power $\zeta_{p}^{k(a, P)}$ for some residue class $k(a, P) \bmod p$. Similarly, any $b \in A$ fixing a black vertex $Q$ acts locally like $z \mapsto \zeta_{q}^{l(b, Q)} z$ for some residue class $l(b, Q) \bmod q$. Observe that conjugation does not change multipliers: for all $g \in A$ we have

$$
k\left(g a g^{-1}, g P\right)=k(a, P) \quad \text { and } \quad l\left(g b g^{-1}, g Q\right)=l(b, Q) .
$$

As shown in the proof of Lemma 5 , the automorphisms of $X$ are birational morphisms defined over $\overline{\mathbf{Q}}$, and their fixed points are $\overline{\mathbf{Q}}$-rational points of $X$. In the following, we will in 
fact identify $A$ with $A^{\sigma}$ by using the isomorphism $A \rightarrow A^{\sigma}, a \mapsto a^{\sigma}$ described in Lemma 5 . The multipliers at these points satisfy

Definition 2. Let $A$ be the automorphism group of a regular dessin $\mathcal{D}$. For each $\sigma$ in the absolute Galois group $\mathrm{Gal} \overline{\mathbf{Q}} / \mathbf{Q}$ we can identify $A$ and $A^{\sigma}$ in the obvious way, so that $A$ is regarded as the automorphism group of $\mathcal{D}^{\sigma}$. We call $\mathcal{D}$ and $\mathcal{D}^{\sigma}$ equivariant for $A$ if there is a colour-preserving isomorphism $f: \mathcal{C} \rightarrow \mathcal{C}^{\prime}$ of the underlying graphs of $\mathcal{D}$ and $\mathcal{D}^{\sigma}$ commuting with the actions of $A$ and changing the multipliers in a uniform way. More precisely, we require that:

(i) $f \circ a=a \circ f$ for all automorphisms $a \in A$;

(ii) there is an integer $s$ coprime to the least common multiple $m$ of $p$ and $q$ such that $k(a, f(P)) \equiv \operatorname{sk}(a, P) \bmod m$ for all $a \in A$ and all fixed points $P$ of $a$.

We then call $f$ an $A$-equivariant graph isomorphism. We say that $\mathcal{D}$ is Galois compatible if $\mathcal{D}$ and $\mathcal{D}^{\sigma}$ are equivariant for $A$ for each $\sigma \in \mathrm{Gal} \overline{\mathbf{Q}} / \mathbf{Q}$.

Lemmas 6 and 7 and equation (2) immediately imply the following.

Proposition 2. If $\mathcal{D}$ is a regular dessin with automorphism group $A$, and the Galois conjugation $\sigma: \mathcal{D} \rightarrow \mathcal{D}^{\sigma}$ is adjacency preserving, then $\mathcal{D}$ and $\mathcal{D}^{\sigma}$ are equivariant for $A$.

Theorem 1. Let $X$ be a quasiplatonic surface with a regular dessin $\mathcal{D}$ of type $(p, q, r)$. As a smooth projective algebraic curve, let $X$ be defined over a subfield of the cyclotomic field $\mathbf{Q}\left(\zeta_{m}\right)$, with $\zeta_{m}:=\exp (2 \pi i / m)$, where $m$ is the least common multiple of $p$ and $q$. Let $\sigma \in \mathrm{Gal} \mathbf{Q}\left(\zeta_{m}\right) / \mathbf{Q}$, so that $\sigma\left(\zeta_{m}\right)=\zeta_{m}^{s}$ for some $s$ coprime to $m$. If there is an A-equivariant graph isomorphism $f$ between the graphs underlying $\mathcal{D}$ and $\mathcal{D}^{\sigma}$, where $A=$ Aut $\mathcal{D}$, then the Galois conjugate dessin $\mathcal{D}^{\sigma}$ results from $\mathcal{D}$ by an application of Wilson's operation $H_{j}$, where $j s \equiv 1 \bmod m$, and hence $\mathcal{D}^{\sigma} \cong H_{j} \mathcal{D}$.

Proof. We use the terminology and the results of Lemmas 4-7, in particular the isomorphism $f: \mathcal{C} \rightarrow \mathcal{C}^{\prime}$ between the graphs underlying $\mathcal{D}$ and $\mathcal{D}^{\sigma}$, and the generation of the automorphism group $A=$ Aut $\mathcal{D}$ by $a_{0}$ and $b_{0}$ as described above.

The dessin $\mathcal{D}$ is uniquely determined by $\mathcal{C}$ and the anticlockwise cyclic rotations of edges around its vertices. The rotation around $P_{0}$ is given by $\left(e_{0}, a_{0} e_{0}, a_{0}^{2} e_{0}, \ldots, a_{0}^{p-1} e_{0}\right)$, where $e_{0}$ is an edge incident with $P_{0}$, and since $\mathcal{D}$ is regular, the rotation around any white vertex $P$ is given by applying successive powers of an automorphism $a$ fixing $P$ and conjugate in $A$ to $a_{0}$. Similarly, the rotation around a black vertex $Q$ is given by successive powers of an automorphism $b$ fixing $Q$ and conjugate to $b_{0}$. Identifying via $f$, we can regard the $\operatorname{dessin} \mathcal{D}^{\sigma}$ as having the same graph $\mathcal{C}$ as $\mathcal{D}$, but possibly with a different local ordering of the edges around the vertices, corresponding to different generating rotations $a$ and $b$ in $A$ fixing them. 
Now $a_{0}$ acts on $\mathcal{D}^{\sigma}$ by fixing $f\left(P_{0}\right)$ (which we have identified with $P_{0}$ ), and by the definition of $A$-equivariance it acts locally around this vertex as multiplication by $\zeta_{p}^{s}$. Thus if we were to choose $a$ conjugate to $a_{0}$, then the resulting cyclic order of edges of $\mathcal{D}^{\sigma}$ around each white vertex $P$ would be given locally by multiplication by $\zeta_{p}^{s}$. Instead, in order to obtain the correct anticlockwise cyclic order of edges around $P$, we need to multiply by $\zeta_{p}$, and therefore we must choose $a$ conjugate to $a_{0}^{j}$, where $j s \equiv 1 \bmod m$. Of course, the same idea applies to the black vertices, with $b$ conjugate to $b_{0}^{j}$. By Lemma 4 , this is equivalent to replacing $\mathcal{D}$ with $H_{j} \mathcal{D}$.

REMARK 4. If $\mathcal{C}^{\prime} \not \mathcal{C}$, then we cannot have $\mathcal{D}^{\sigma} \cong H_{j} \mathcal{D}$ for any $j$ since $H_{j} \mathcal{D}$ has the same embedded graph $\mathcal{C}$ as $\mathcal{D}$, whereas $\mathcal{D}^{\sigma}$ has $\mathcal{C}^{\prime}$ as its embedded graph. See Example 8 in Section 4 and Corollary A.1 in the Appendix.

Theorem 2. Let $X_{j}$, with $j \in(\mathbf{Z} / m \mathbf{Z})^{*}$, denote a family of quasiplatonic surfaces of type $(p, q, r)$, where $m$ denotes the least common multiple of $p$ and $q$, with regular dessins $\mathcal{D}_{j}:=$ $H_{j} \mathcal{D}_{1}$ forming an orbit under Wilson's map operations $H_{j}$ and corresponding to Belyu functions $\beta_{j}$ on $X_{j}$. Suppose that the family of pairs $\left\{\left(X_{j}, \beta_{j}\right), j \in(\mathbf{Z} / m \mathbf{Z})^{*}\right\}$ is invariant under the action of the absolute Galois group Gal $\overline{\mathbf{Q}} / \mathbf{Q}$. Then, as smooth projective algebraic curves, the curves $X_{j}$ and their Belyı̆ functions $\beta_{j}$ can be defined over a subfield $K$ of the cyclotomic field $\mathbf{Q}\left(\zeta_{m}\right)$, and they form a single orbit under the action of the absolute Galois group. Here, $K$ is the fixed field of the subgroup

$$
H:=\left\{j \in(\mathbf{Z} / m \mathbf{Z})^{*} \mid H_{j} \mathcal{D}_{1} \cong \mathcal{D}_{1}\right\},
$$

where we identify $(\mathbf{Z} / m \mathbf{Z})^{*}$ with the Galois group Gal $\mathbf{Q}\left(\zeta_{m}\right) / \mathbf{Q}$.

Proof. Suppose that $\sigma \in \mathrm{Gal} \overline{\mathbf{Q}} / \mathbf{Q}$ is a Galois conjugation with $X_{1}^{\sigma} \cong X_{j}$ and $\mathcal{D}_{1}^{\sigma} \cong \mathcal{D}_{j}$. Equation (2) also applies in this case to all multipliers. Therefore, if $a \in A$ generates the stabiliser of the white vertex $P$ of $\mathcal{D}_{1}$, with multiplier $\zeta_{p}$, it fixes $P^{\sigma}$ with multiplier $\sigma\left(\zeta_{p}\right)$. By definition of $H_{j}$ (see Lemma 1), $a^{j}$ has the correct multiplier $\zeta_{p}$ as a generator of the stabiliser $P^{\sigma}$, and hence $\sigma\left(\zeta_{p}^{j}\right)=\zeta_{p}$. The situation is completely analogous for the black vertices, and so for all $\sigma$ fixing the cyclotomic field $\mathbf{Q}\left(\zeta_{m}\right)$ elementwise, we have $\mathcal{D}_{1}^{\sigma} \cong \mathcal{D}_{1}$, and hence $X_{1}^{\sigma} \cong X_{1}$. Moreover, this is the case for each $\sigma$ whose restriction to $\mathbf{Q}\left(\zeta_{m}\right)$ belongs to $H$. This means that the moduli field of $\left(X_{1}, \beta_{1}\right)$ is contained in the cyclotomic field $K \subseteq \mathbf{Q}\left(\zeta_{m}\right)$ corresponding to $H$, and by [4] (see also [8], [28, Remark 4] or [29, Theorem 5]), $X_{1}$ and $\beta_{1}$ can be defined over this moduli field. On the other hand, any $\sigma \in \mathrm{Gal} \mathbf{Q}\left(\zeta_{m}\right) / \mathbf{Q}$ with $\sigma\left(\zeta_{m}\right)=\zeta_{m}^{s}$ sends $\mathcal{D}_{1}$ to a Galois conjugate dessin $\mathcal{D}_{1}^{\sigma}$ which, by hypothesis, belongs to the family $\left\{\mathcal{D}_{j}\right\}$. The behaviour at the fixed vertices again shows that $j$ must satisfy $s j \equiv 1 \bmod m$.

REMARK 5. Since $H_{i} H_{j}=H_{j} H_{i}$ for all $i$ and $j$, we can use any $\mathcal{D}_{i}$ instead of $\mathcal{D}_{1}$ in the definition of $H$.

REMARK 6. Since we are considering isomorphisms of dessins, and not just curves, it follows that $K$ is in fact the minimal field of definition of a Bely̌ pair $\left(X_{j}, \beta_{j}\right)$, where $\beta_{j}$ is the Belyı function corresponding to $\mathcal{D}_{j}$. It may happen that the moduli field (minimal field of definition) of $X_{j}$ is smaller (see [22, Remark 1]), if there are isomorphisms $X_{j} \cong X_{k}$ not compatible with the corresponding Belyı̆ functions. 
If we consider the white and the black vertices separately in the proof of Theorem 2 , then we get an obvious extension to Wilson's generalised operations $H_{i, j}$ introduced at the end of Section 2.

Theorem 3. Let $X_{i, j}$, with $(i, j)$ running over a subset $S \subseteq(\mathbf{Z} / p \mathbf{Z})^{*} \times(\mathbf{Z} / q \mathbf{Z})^{*}$, denote a family of quasiplatonic surfaces of type $(p, q, r)$. Let $m$ denote the least common multiple of $p$ and $q$, and suppose that $S$ admits an action of $(\mathbf{Z} / m \mathbf{Z})^{*}$, that is whenever $S$ contains $(i, j)$ it also contains $(k i, k j)$ for all $k \in(\mathbf{Z} / m \mathbf{Z})^{*}$. Suppose that these curves $X_{i, j}$ are equipped with regular dessins $\mathcal{D}_{i, j}:=H_{i, j} \mathcal{D}_{1,1}$ forming an orbit under Wilson's generalised operations $H_{i, j}$ and corresponding to Belyy functions $\beta_{i, j}$ on $X_{i, j}$. Suppose that the family of pairs $\left\{\left(X_{i, j}, \beta_{i, j}\right),(i, j) \in S\right\}$ is invariant under the action of the absolute Galois group Gal $\overline{\mathbf{Q}} / \mathbf{Q}$. Then, as smooth projective algebraic curves, the curves $X_{i, j}$ and their Belyu functions $\beta_{i, j}$ can

470 be defined over a subfield $K(i, j)$ of the cyclotomic field $\mathbf{Q}\left(\zeta_{m}\right)$. The orbits of the absolute Galois group consist of the dessins as follows:

$$
H_{k i, k j} \mathcal{D}_{1,1}=H_{k} \circ H_{i, j} \mathcal{D}_{1,1}, \quad k \in(\mathbf{Z} / m \mathbf{Z})^{*},
$$

and the minimal field of definition of the Belyı pair $\left(X_{i, j}, \beta_{i, j}\right)$ is the fixed field $K(i, j) \subseteq \mathbf{Q}\left(\zeta_{m}\right)$ of all $k \in(\mathbf{Z} / m \mathbf{Z})^{*}$ with the property that $\mathcal{D}_{i, j} \cong \mathcal{D}_{k i, k j}$.

Proof. By the same multiplier arguments as in the proof of Theorem 2, all the dessins $\mathcal{D}_{i, j}$ in question are invariant under Gal $\overline{\mathbf{Q}} / \mathbf{Q}\left(\zeta_{m}\right)$, and algebraic conjugations within $\mathbf{Q}\left(\zeta_{m}\right)$ act like Wilson's operations $H_{k}$.

\section{Known Galois orbits}

We shall examine the next example in some detail, in order to illustrate the general principles underlying the preceding theorems.

ExAmple 4. Suppose that $p$ is a prime and $q$ an integer greater than 2 dividing $p-1$, and let $A$ be the semidirect product of a cyclic normal subgroup $\langle a\rangle$ of order $p$ by a cyclic subgroup $\langle b\rangle$ of order $q$ acting on $\langle a\rangle$ by $b^{-1} a b=a^{u}$, where the integer $u$ represents a fixed residue class of order $q$ in $(\mathbf{Z} / p \mathbf{Z})^{*}$ (the isomorphism class of $A$ is independent of the choice of this class).

${ }_{485}$ Along the lines of [22, Sections 2 and 3], one can prove that there are precisely $\phi(q)$ nonisomorphic regular dessins $\mathcal{D}(s)$, with $s \in(\mathbf{Z} / q \mathbf{Z})^{*}$, of type $(p, q, q)$ with automorphism group $A$, determined by the generators $h\left(\gamma_{0}\right)=a_{0}=a$ and $h\left(\gamma_{1}\right)=b_{0}=b^{s}$. (In [22] more restrictive assumptions on $p$ and $q$ were made, which are not necessary for the present paper; see, for example, [22, Remark 2].) These dessins $\mathcal{D}(s)$ are all cyclic coverings of a common quotient $\mathcal{M}=\mathcal{D}(s) /\langle a\rangle$, a genus 0 dessin with one white vertex of valency $q$ at $x=0$ and $q$ black vertices of valency 1 at $x=\zeta_{q}^{j}, j \bmod q$, corresponding to the Belyu function $x^{q}$. Each $\mathcal{D}(s)$ is ramified of order $p$ at all the black vertices of $\mathcal{M}$, so that the embedded graph of $\mathcal{D}(s)$ is the complete bipartite graph $K_{p, q}$.

We use this example first for an application of Theorem 2. Since type and automorphism group are Galois invariants, these dessins form a Galois invariant family. Then we can modify the homomorphism $h$ without changing the kernel by defining

$$
h\left(\gamma_{0}\right)=a_{0}=a^{s}, \quad h\left(\gamma_{1}\right)=b_{0}=b^{s}
$$

for any $s$ prime to $p q$ (the dessin still depends only on $s$ mod $q$ and one may assume that $s \equiv 1 \bmod p$ ), and thus by Lemma 4 we have $\mathcal{D}(s)=H_{s} \mathcal{D}(1)$. Hence Theorem 2 implies that 
Theorem 1]. Specifically, an affine model of the curve $X_{s}$ corresponding to $\mathcal{D}(s)$ is given by

$$
y^{p}=\prod_{j=1}^{q}\left(x-\zeta_{q}^{\bar{s} j}\right)^{u^{j}},
$$

where $\zeta_{q}=\exp (2 \pi i / q)$ and $\bar{s} s \equiv 1 \bmod q$.

Conversely we can use this field of definition and the common graph to see that these Galois conjugate dessins result from each other by Wilson's operations $H_{s}$ : since the graphs are complete, all Galois conjugations are clearly adjacency preserving, and hence Theorem 1 applies.

For completeness, we consider the behaviour of the generating automorphisms around the fixed points. We may take $y$ as a local variable near the $q$ white vertices $P_{i}=\left(\zeta_{q}^{i}, 0\right)$, and $x$ as the local variable near the $p$ black vertices $Q_{j}=\left(0, \zeta_{p}^{j}\right)$. As rational maps, the generating automorphisms are given by

$$
a:(x, y) \longmapsto\left(x, \zeta_{p} y\right), \quad b:(x, y) \longmapsto\left(\zeta_{q}^{\bar{s}} x, \zeta_{p}^{\bar{u}} y^{u}(x-1)^{\left(1-u^{q}\right) / p}\right),
$$

where we define $\bar{u}$ by $\bar{u} u \equiv 1 \bmod p$. One can check the relation $a b=b a^{u}$ and verify that $a^{s}$ and $b^{s}$ have the correct multipliers (recall that $s \equiv 1 \bmod p$ ).

EXAmple 5. Now we consider the same group $A$ as in Example 4 under the more restrictive assumptions that $p$ and $q$ are both primes greater than 3 with $p \equiv 1 \bmod q$ and $q \neq 7$. The group $A$ also occurs in [22, Corollary 1] as the automorphism group of $(q-1)(q-2)$ regular dessins $\mathcal{D}(n, t)$ of type $(q, q, q)$ for all $n, t \in(\mathbf{Z} / q \mathbf{Z})^{*}$ with $n+t \not \equiv 0 \bmod q$, in this case with $h\left(\gamma_{0}\right):=b^{n}$, and $h\left(\gamma_{1}\right):=a b^{t}$. We can replace these generators with

$$
a_{0}=b^{n}, \quad b_{0}=(a b)^{t}
$$

by composing $h$ with an automorphism of $A$; see [22, Lemma 3]. With these generators, Lemma 4 applies and shows that for Wilson's generalised operations $H_{n, t} \mathcal{D}(1,1)=\mathcal{D}(n, t)$ for all $n$ and $t$ coprime to $q$ with $n+t \not \equiv 0 \bmod q$. It is shown in [22, Theorem 3] that these dessins are all defined over $\mathbf{Q}\left(\zeta_{q}\right)$, and that for fixed $n$ and $t$ the dessins $\mathcal{D}(k n, k t)$, with $k \in(\mathbf{Z} / q \mathbf{Z})^{*}$, form a single Galois orbit. With $S:=\left\{(n, t) \in\left((\mathbf{Z} / q \mathbf{Z})^{*}\right)^{2} \mid n+t \not \equiv 0 \bmod q\right\}$, this is now a direct application of Theorem 3 since Galois conjugation leaves invariant the automorphism group and ramification properties. Thus these dessins $\mathcal{D}(n, t)$ in fact form a Galois invariant family.

On the other hand, knowing that the corresponding Belyı pairs are defined over $\mathbf{Q}\left(\zeta_{q}\right)$, we can also apply Theorem 1 to them, provided, for instance, we can show that Galois conjugation is adjacency preserving on the dessins $\mathcal{D}(n, t)$. To do so, we remark that these dessins all have the same graph: if we label the white and black vertices with $\mathbf{F}_{p}$ so that $A$ acts as a subgroup of $\operatorname{AGL}_{1}\left(\mathbf{F}_{p}\right)$, then $P_{i}$ is joined to $Q_{j}$ if and only if $j-i$ is in a specific coset of $\langle u\rangle$ depending on $u$. (For a given $p$ and $q$, all such graphs are isomorphic, and so the choice of a generator $u$ is unimportant.) In particular, $a$ generates translations on the sets of white and black vertices (without fixed points), and the elements $b$ and $a b$ have precisely one fixed point each on the respective sets of white and black vertices. If we think of Galois conjugations as identifying the automorphism group and preserving the labelling of the vertices, then neighbouring vertices $P_{i}$ and $Q_{j}$ (as the unique white and black fixed points of $b$ and $a b$ ) must remain neighbouring fixed points under the Galois action because $j-i$ remains invariant. Thus Proposition 2 and Theorem 1 apply, showing that Galois conjugacy implies Wilson conjugacy.

More generally, if we apply $H_{i, j}$, replacing $a_{0}$ and $b_{0}$ with $a_{0}^{i}$ and $b_{0}^{j}$ for arbitrary $i$ and $j$ both coprime to $q$, then we obtain all $(q-1)(q-2)$ dessins of this type $(q, q, q)$, together with $q-1$ dessins of type $(q, q, p)$ where $n+t \equiv 0 \bmod q$; these are the duals of the dessins in Example 4 , interchanging white vertices and face centres. 
EXAMPLE 6. Let $n=p^{e}$ be an odd prime power and let $\mathcal{M}$ be a regular dessin based on an embedding of the complete bipartite graph $\mathcal{B}=K_{n, n}$ such that the map, and not just the hypermap, is regular. These dessins have been classified in [13]: they are of type $(n, n, n)$ with the automorphism group (of the hypermap) as follows:

$$
G_{f}:=\left\langle g, h \mid g^{n}=h^{n}=1, h^{g}=h^{1+p^{f}}\right\rangle \quad(f \in\{1, \ldots, e\}) .
$$

Their isomorphism classes are $\mathcal{M}(f ; u)$, where $u \in\left(\mathbf{Z} / p^{e-f} \mathbf{Z}\right)^{*}$, the different dessins being distinguished by the choice of a pair of generators $a_{0}=g^{u}$ and $b_{0}=(g h)^{u}$; see $[\mathbf{1 3}$, Theorem 1 ; 16, Lemma 1]. Here one has to take a representative $u \bmod n$, but the isomorphism class of the dessin depends only on the residue class $u \bmod p^{e-f}$. It follows from Lemma 4 (see also [13]) that for each fixed $f$ the maps $\mathcal{M}(f ; u)$ form a single orbit under Wilson's operations, with $H_{j} \mathcal{M}(f ; u) \cong \mathcal{M}(f ; j u)$ for all $j$ coprime to $p$; Theorems 1 and 2 of [16] show that they correspond to Belyı pairs defined over $\mathbf{Q}\left(\zeta_{p^{e-f}}\right)$, and that they form a single Galois orbit. Since all graphs are complete bipartite, adjacency is preserved under Galois conjugation, and hence these results again illustrate Theorems 1 and 2 .

ExAMPLE 7. Once again consider a regular embedding of $K_{n, n}$, with $n=p^{e}$ an odd prime power, but now regular only as a hypermap (that is with an edge-transitive automorphism group which is not necessarily transitive on directed edges). We again get hypermaps of type $(n, n, n)$ with automorphism group $G_{f}$ as in (3), but now many more pairs of generators are allowed. Up to a permutation of the three generators of $\Delta=\langle n, n, n\rangle$ (that is exchange of black vertices, white vertices and face centres), we can take them to be

$$
a_{0}=g^{u}, \quad b_{0}=(g h)^{v}, \quad u, v \in(\mathbf{Z} / n \mathbf{Z})^{*} .
$$

As before, the isomorphism classes of dessins $\mathcal{M}(f ; u, v)$ depend only on $u, v \bmod p^{e-f}$, and by Lemma 4 we again have

$$
\mathcal{M}(f ; i u, j v) \cong H_{i, j} \mathcal{M}(f ; u, v) \text { for all } i, j \text { coprime to } p .
$$

This series of dessins is also Galois invariant because they are, up to the permutations of the generators mentioned above, the only ones sharing regularity, type and automorphism group $G_{f}$. Therefore Theorem 3 applies and shows that the family $\mathcal{M}(f ; u, v)$ splits into Galois orbits $\mathcal{M}(f ; k u, k v)$, where $k \in(\mathbf{Z} / n \mathbf{Z})^{*}$. Theorem 2 applies to these orbits. For more detailed information, in particular the fields of definition of the underlying curves and their equations; see [6]. Since all graphs are again complete bipartite, adjacency is also preserved in these cases.

EXAMPLE 8. The preceding examples may give the impression that dessins that are Galois conjugate are always equivalent under Wilson's operations. The following example shows that this is not always the case.

A curve $X$ of genus $g>1$ is a Hurwitz curve if $\mid$ Aut $X \mid$ attains Hurwitz's upper bound of $84(g-1)$. This is equivalent to $X$ being uniformised by a normal subgroup $\Gamma$ of finite index $|\Delta: \Gamma|>1$ in the triangle group $\Delta$ of signature $(7,2,3)$, and hence such surfaces are quasiplatonic and correspond to regular dessins of type $(7,2,3)$, that is, regular maps of type $(7,3)$.

Macbeath [19] showed that for each prime $p \equiv \pm 1 \bmod 7$ there are three Hurwitz curves, known as Macbeath-Hurwitz curves, with an automorphism group $\mathrm{PSL}_{2}\left(\mathbf{F}_{p}\right)$. As shown in [21], they form a family of three Galois conjugate curves, defined over the cubic field $\mathbf{Q}\left(\cos \frac{2 \pi}{7}\right) \subset$ $\mathbf{Q}\left(\zeta_{7}\right)$. In the generating triples $(x, y, z)$ for the corresponding dessins the elements $x$ lie in the three conjugacy classes of elements of order 7 in $\mathrm{PSL}_{2}\left(\mathbf{F}_{p}\right)$, distinguished from each other by their traces. In fact, not only are these three curves and their corresponding dessins mutually non-isomorphic, but so are their underlying graphs, as shown in the Appendix. 
There is another reason as well showing that Galois conjugations and Wilson's operations do not coincide on Macbeath-Hurwitz curves: Wilson's operations may change the valencies of the faces of the maps. The case $p=13$ is simple and instructive. The elements of order 7 in $\operatorname{PSL}_{2}\left(\mathbf{F}_{13}\right)$ consist of the three classes $C(t)$ of elements with traces $t= \pm 3, \pm 6$ and \pm 5 , permuted in that cyclic order by squaring. The corresponding dessins $\mathcal{D}(t)$ are distinguished from each other by the order of the commutator $c=[x, y]=x^{-1} y^{-1} x y$, which is 7,6 or 13 , so that the Petrie polygons in these maps have lengths 14,12 or 26 respectively. (This example shows that Petrie length, unlike various other combinatorial parameters such as valencies of vertices and faces, is not Galois invariant.) We can apply Wilson's operation $H_{2}$ to each map $\mathcal{D}(t)$, replacing $x$ with $x^{\prime}=x^{2}$ but using the same $y$, or equivalently we can apply $H_{9}$ to the Walsh map, sending $x$ to $x^{9}=x^{2}$ and $y$ to $y^{9}=y$, so that $z=(x y)^{-1}$ is replaced with $z^{\prime}=\left(x^{2} y\right)^{-1}=y x^{5}$. In all the three cases $z^{\prime}$ has order 7 rather than 3 , and thus we get three non-isomorphic regular maps $H_{2} \mathcal{D}(t)$ of type $(7,7)$, with $x^{\prime}$ and $z^{\prime}$ in classes $C(6)$ and $C(3)$, $C(5)$, and $C(6)$, and $C(3)$ and $C(5)$, respectively. However, if we apply the inverse operation $H_{4}$ to each $D(t)$, then we get maps $H_{4} \mathcal{D}(t)$ of three different types, namely, type $(7,7)$ with $x^{\prime}, z^{\prime} \in C(5)$, type $(7,6)$ with $x^{\prime} \in C(3)$ and type $(7,13)$ with $x^{\prime} \in C(6)$.

The situation is similar for the next possible prime $p=29$. In this case the elements of order 7 have traces $\pm 3, \pm 7$ and \pm 11 , and are permuted by squaring in this cyclic order. The commutator $c=[x, y]$ has trace $\pm 8, \pm 10$ and \pm 4 respectively, so that its order is 15,14 and 15 giving Petrie lengths 30,28 and 30 respectively. This distinguishes the second map from the first and third, and for a combinatorial distinction between these two we can consider the element $x^{4} y x^{2} y$, representing a generalised Petrie path turning alternately fourth and second right: in these two cases it has order 29 and 14, giving path-lengths 58 and 28 respectively. Applying $H_{2}$ to any of these three maps gives a map of type $(7,7)$, with $z^{\prime}$ conjugate to $x$, whereas applying $H_{4}$ gives maps of types $(7,15),(7,14)$ and $(7,15)$, with $z^{\prime}$ conjugate to $c$ in all cases. Once again we have a Galois orbit that is not an orbit under Wilson's operations.

To conclude this section, we recall the common ideas behind the earlier papers that considered the Galois actions on the families of dessins in Examples 4-8. It is unclear whether this method has a common graph-theoretic interpretation, but its advantage is the possible application to additional examples such as the Macbeath-Hurwitz curves, where no graph isomorphism is available.

Definition 3. Let $\mathcal{D}$ be a regular dessin of type $(p, q, r)$, and let its automorphism group $A$ have canonical generators $a_{0}$ and $b_{0}$. Suppose that for each $i \in(\mathbf{Z} / p \mathbf{Z})^{*}, a_{0}$ fixes $l_{i}$ white vertices of $\mathcal{D}$ with multiplier $\zeta_{p}^{i}$, that for each $j \in(\mathbf{Z} / q \mathbf{Z})^{*}, b_{0}$ fixes $m_{j}$ black vertices with multiplier $\zeta_{q}^{j}$, and that for each $k \in(\mathbf{Z} / r \mathbf{Z})^{*}, a_{0} b_{0}$ fixes $n_{k}$ face centres with multiplier $\zeta_{r}^{k}$. The sets of all such pairs

$$
\left(i, l_{i}\right) \in(\mathbf{Z} / p \mathbf{Z})^{*} \times \mathbf{N}_{0}, \quad\left(j, m_{j}\right) \in(\mathbf{Z} / q \mathbf{Z})^{*} \times \mathbf{N}_{0}, \quad\left(k, n_{k}\right) \in(\mathbf{Z} / r \mathbf{Z})^{*} \times \mathbf{N}_{0},
$$

which are independent of the choice of $a_{0}, b_{0}$ by the conjugacy of canonical generating pairs, are called the multiplier data for $\mathcal{D}$.

Using formula (2) again one can easily see how the absolute Galois group induces an action on the multiplier data: each $\sigma$ acts on roots of unity $\zeta$ of orders $p, q$ and $r$ as $\sigma(\zeta)=\zeta^{s}$ for some $s$ coprime to $p q r$, so that the multiplier data for $\mathcal{D}^{\sigma}$ are obtained from those for $\mathcal{D}$ by replacing the above sets of pairs with the sets of pairs $\left(s i, l_{i}\right),\left(s j, m_{j}\right)$ and $\left(s k, n_{k}\right)$ respectively. 
Proposition 3. Let $A$ be a finite group and let $\mathcal{F}$ be the family of all regular dessins $\mathcal{D}$ of type $(p, q, r)$ with automorphism group $A$. Suppose that there exists a bijection between $\mathcal{F}$ and the multiplier data for its dessins $\mathcal{D}$. Then these dessins are all defined over the cyclotomic field $\mathbf{Q}\left(\zeta_{p}, \zeta_{q}, \zeta_{r}\right)$, and the orbits of the absolute Galois group on $\mathcal{F}$ are in one-to-one correspondence with its orbits on the multiplier data.

Proof. As in the proof of Theorem 2, the type and automorphism group of a dessin are Galois invariant, so $\mathcal{F}$ is a Galois invariant family. If $\sigma$ fixes $\mathbf{Q}\left(\zeta_{p}, \zeta_{q}, \zeta_{r}\right)$ elementwise, then it fixes all the multiplier data. Since the dessins in $\mathcal{F}$ are uniquely determined by their multiplier data, they are invariant under those $\sigma$ and are therefore defined over a subfield of $\mathbf{Q}\left(\zeta_{p}, \zeta_{q}, \zeta_{r}\right)$. The precise field of moduli of a dessin, equal to its minimal field of definition, is determined by its Galois orbit, and hence by the corresponding Galois orbit on the multiplier data.

\section{Regular dessins based on complete graphs}

By the work of James and the first author of the present paper [12] it is known that there are regular maps whose underlying graph is the complete graph $K_{n}$ if and only if $n$ is a prime power $p^{e}$. The regular embeddings of $K_{n}$ were previously constructed by Biggs [3] in the following way. Label the vertices with the elements of the finite field $\mathbf{F}_{n}$, choose a generator $u$ of the cyclic multiplicative group $\mathbf{F}_{n}^{*}$ and join each vertex $v \in \mathbf{F}_{n}$ by an edge to each of the other vertices in the anticlockwise cyclic order

$$
v+1, v+u, v+u^{2}, \ldots, v+u^{n-2}
$$

around $v$. This ordering defines a regular map $\mathcal{M}(n, u)$. Considered as a hypermap, it is of type $(n-1,2, r)$ with

$$
r=\frac{1}{2}(n-1) \text { for } 3<n \equiv 3 \bmod 4 \text { and } r=n-1 \text { for other } n>3
$$

on a Riemann surface $X(n, u)$ of genus $\left(n^{2}-7 n+4\right) / 4$ if $n \equiv 3 \bmod 4$ and $(n-1)(n-4) / 4$ for all other $n>3$ (see [12, p. 362]). The automorphism group of $\mathcal{M}(n, u)$ is the affine group $\mathrm{AGL}_{1}\left(\mathbf{F}_{n}\right)$, acting naturally on the vertices. Two such dessins $\mathcal{M}(n, u)$ and $\mathcal{M}\left(n, u^{\prime}\right)$ are isomorphic if and only if $u$ and $u^{\prime}$ belong to the same orbit under the action of the Galois group Gal $\mathbf{F}_{n} / \mathbf{F}_{p}$ (see [12, Theorem B]). Since this group is cyclic of order $e$, generated by the Frobenius automorphism $u \mapsto u^{p}$, there are $\phi(n-1) / e$ maps $\mathcal{M}(n, u)$ up to isomorphism. It is clear from the construction of these maps that $H_{j} \mathcal{M}(n, u)=\mathcal{M}\left(n, u^{j}\right)$ for all $j$ coprime to $n-1$ (see [12, p. 362]), thus illustrating Corollary 1 and also showing that for fixed $n$ they are all equivalent under Wilson's operations. Theorem 2 implies the following.

TheOREM 4. Let $n$ be a prime power $p^{e}$ and let $\left\{\mathcal{M}(n, u) \mid u \in(\mathbf{Z} /(n-1) \mathbf{Z})^{*}\right\}$ be the family of regular maps resulting from all regular embeddings of the complete graph $K_{n}$ into orientable surfaces $X(n, u)$, equipped with the structure of smooth complex projective algebraic curves over number fields and with the regular Belyu functions $\beta_{u}$ corresponding to the dessins $\mathcal{M}(n, u)$. Then these Belyı pairs $\left(X(n, u), \beta_{u}\right)$ form a single Galois orbit. Their minimal field of definition is the splitting field $K$ of the prime $p$ in the cyclotomic field extension $\mathbf{Q}\left(\zeta_{n-1}\right) / \mathbf{Q}$.

Proof. Since regularity is Galois invariant [15] and since from [12] we have a complete list of regular maps based on $K_{n}$, our family is Galois invariant, and hence Theorem 2 applies: Wilson's operation $H_{j}$ replaces $u$ with $u^{j}$ in the above definition of the cyclic order around the vertices. The only remaining point is to determine the field of definition $K$. Again by Theorem 2 
and $[\mathbf{1 2}], K$ is the fixed field of the subgroup

$$
H=\left\{p^{k} \bmod n-1 \mid k=1, \ldots, e\right\} \leqslant(\mathbf{Z} /(n-1) \mathbf{Z})^{*} \cong \operatorname{Gal} \mathbf{Q}\left(\zeta_{n-1}\right) / \mathbf{Q}
$$

inducing all residue class Galois groups for all prime ideals extending $p$ (unramified in $\mathbf{Q}\left(\zeta_{n-1}\right)$ since $p$ is coprime to $n-1)$. Therefore $K$ is the splitting field of $p$, that is the maximal subextension of $\mathbf{Q}\left(\zeta_{n-1}\right) / \mathbf{Q}$ in which $p$ decomposes completely into $d$ different prime ideals of degree 1 over $p$, where $d=[K: \mathbf{Q}]=\phi(n-1) / e$.

EXAmple 9. Let $n=8$. Then $p=2, e=3$ and there are $d=\phi(7) / 3=2$ regular maps $\mathcal{M}(8, u)$. If we take $\mathbf{F}_{8}=\mathbf{F}_{2}[x] /\left(x^{3}+x+1\right)$, then these two maps correspond to choosing $u$ from the subsets $\left\{x, x^{2}, x^{4}=x^{2}+x\right\}$ or $\left\{x+1,(x+1)^{2}=x^{2}+1,\left(x^{2}+1\right)^{2}=x^{2}+x+1\right\}$ of $\mathbf{F}_{8}^{*}$. These two sets are mutually inverse in $\mathbf{F}_{8}$, and hence the two maps form a chiral (mirror-image) pair $\mathcal{M}, H_{-1} \mathcal{M}$, namely, the Edmonds maps of genus 7 . If $\zeta:=\zeta_{7}$ and $\eta:=\zeta+\zeta^{2}+\zeta^{4}=(-1+\sqrt{-7}) / 2$, then the two corresponding Belyı pairs are defined over the field $K=\mathbf{Q}(\eta)=\mathbf{Q}(\sqrt{-7})$ fixed by the automorphism $\zeta \mapsto \zeta^{2}$ of order 3 of the cyclotomic field $\mathbf{Q}(\zeta)$, and they are conjugate under its automorphism $\zeta \mapsto \zeta^{-1}$, or equivalently $\sqrt{-7} \mapsto-\sqrt{-7}$. The decomposition of $p=2$ in this field is given by $2=-\eta(\eta+1)$.

In this example, considered as a hypermap with black vertices as midpoints of all edges in $K_{n}$, it is obvious that Galois conjugation preserves adjacency between the white and black vertices of valencies $n-1$ and 2 , since it acts as a reflection. In other instances of Theorem 4 , it is unclear whether adjacency is always preserved. For instance, when $n=25$ there are $\phi(24) / 2=4$ regular maps, forming two chiral pairs: if $u$ is a primitive element of $\mathbf{F}_{25}$, then one pair corresponds to $u^{ \pm 1}$ and the other to $u^{ \pm 7}$. Here Wilson's operations and the absolute Galois group both act on these dessins as the Klein four-group $\{ \pm 1, \pm 7\}$ in $(\mathbf{Z} / 24 \mathbf{Z})^{*}$, while the complementary subgroup $\{1,5\}$ (corresponding to the Galois group of $\mathbf{F}_{25}$ ) acts by dessin automorphisms only. As in the case $n=8$, the Galois conjugations corresponding to $\{ \pm 1\}$ preserve adjacency, but it is unclear whether those corresponding to $\{ \pm 7\}$ also do. However, since all dessins of the family are based on the graph $K_{8}$, it is easy to see that there are always $A$-equivariant graph isomorphisms between them, and thus we could use this weaker hypothesis to apply Theorem 1 in these cases as well.

In [11], James classified the orientable embeddings of $K_{n}$ that are edge-transitive but not regular, so that the automorphism group acts transitively on edges but not on directed edges. Such maps exist if and only if $n$ is a prime power $p^{e}$, with $n \equiv 3 \bmod 4$ and $n>3$. As before, the vertices of such a map are labelled with the elements of $\mathbf{F}_{n}$. Choosing a generator $u$ of $\mathbf{F}_{n}^{*}$ and an odd integer $k$ with $1<k<n-1$, we define a map $\mathcal{M}(n, u, k)$ by joining each vertex $v \in \mathbf{F}_{n}$ by an edge to each of the other vertices in the following anticlockwise cyclic order:

$$
v+1, v+u^{k}, v+u^{2}, v+u^{k+2}, v+u^{4}, v+u^{k+4}, \ldots, v+u^{n-3}, v+u^{k+n-3} .
$$

Two such maps $\mathcal{M}(n, u, k)$ and $\mathcal{M}\left(n, u^{\prime}, k^{\prime}\right)$ are isomorphic if and only if $u$ and $u^{\prime}$ are in the same orbit of $\operatorname{Gal} \mathbf{F}_{n} / \mathbf{F}_{p}$ and $k^{\prime} \equiv k$ or $2-k \bmod n-1$ (see [11, Theorem $\left.\mathrm{B}^{\prime}\right]$ ), and thus there are $(n-3) \phi(n-1) / 4 e$ isomorphism classes of such maps [11, Theorem A].

The automorphism group $A$ of $\mathcal{M}(n, u, k)$ is the unique subgroup of index 2 in $\operatorname{AGL}_{1}\left(\mathbf{F}_{n}\right)$, consisting of the affine transformations $t \mapsto a t+b$ of $\mathbf{F}_{n}$ such that $a$ is a square in $\mathbf{F}_{n}^{*}$. This group acts transitively on the vertices and on the edges of the map, but it has two orbits on the faces, each edge separating faces from different orbits. For instance, the edge joining vertices 0 and $u^{k}$ separates two faces whose stabilisers in $A$ are cyclic groups generated by the affine transformations $a_{0}: t \mapsto-u^{-k} t+1$ and $b_{0}: t \mapsto-u^{k-2} t+u^{k}$, rotating the incident vertices in the anticlockwise cyclic orders $\ldots, u^{k}, 0,1, \ldots$ and $\ldots, u^{2}, 0, u^{k}, \ldots$ The valencies $m_{0}$ and $m_{1}$ of these faces are the orders of $a_{0}$ and $b_{0}$ respectively. Since $-1=u^{(n-1) / 2}$ and $k$ 
is odd, we find that $m_{0}=(n-1) / 2(n-1, k)$ unless $k \equiv(n-1) / 2 \bmod (n-1)$, in which case $m_{0}=p$; similarly $m_{1}=(n-1) / 2(n-1, k-2)$ unless $k-2 \equiv(n-1) / 2 \bmod (n-1)$, in which case $m_{1}=p$. This allows us to count the faces, and hence to compute the genus of the map [11].

The dual map $\mathcal{M}(n, u, k)^{*}$ is bipartite, with white and black vertices corresponding to the two orbits of $A$ on the faces of $\mathcal{M}(n, u, k)$, and thus it is the Walsh map of a hypermap $\mathcal{D}(n, u, k)$ of type $\left(m_{0}, m_{1},(n-1) / 2\right)$. In fact, the product $a_{0} b_{0}: t \mapsto u^{-2} t$ has order $(n-1) / 2$ and rotates the face of $\mathcal{M}(n, u, k)^{*}$ resulting from the vertex $v=0$ of the original map $\mathcal{M}(n, u, k)$. Since $A$ acts transitively on the edges of $\mathcal{M}(n, u, k)^{*}$, this hypermap is regular, corresponding to the algebraic hypermap with automorphism group $A$ and generators $a_{0}, b_{0}$. Observe that the involution $k \mapsto 2-k$ transposes the vertex colours of the hypermap.

Theorem 5. With $n=p^{e} \equiv 3 \bmod 4$, greater than 3 and $u, k, m_{0}$ and $m_{1}$ as above, let

$$
S:=\left\{(i, j) \mid i \in\left(\mathbf{Z} / m_{0} \mathbf{Z}\right)^{*}, j \in\left(\mathbf{Z} / m_{1} \mathbf{Z}\right)^{*}, j(k-2)-i k \text { is coprime to } \frac{n-1}{2}\right\} .
$$

For these $(i, j) \in S$, we have an isomorphism $H_{i, j} \mathcal{M}(n, u, k)^{*} \cong \mathcal{M}\left(n, u^{s}, k^{\prime}\right)^{*}$ with $s \equiv \frac{1}{2}[i k-j(k-2)] \bmod ((n-1) / 2)$ and $k^{\prime} \equiv i k / s \bmod n-1$ if we assume $i, j, s$ to be represented by odd integers. They form a Galois invariant family of dessins. If we identify dessins resulting from each other by transposition of the vertex colours, then this family splits into $(n-3) / 4$ Galois orbits

$$
\left\{H_{r i, r j} \mathcal{M}(n, u, k)^{*}, r \in(\mathbf{Z} /(n-1) \mathbf{Z})^{*}\right\}=\left\{\mathcal{M}\left(n, u^{s}, k\right)^{*}, s \in(\mathbf{Z} /(n-1) \mathbf{Z})^{*}\right\}
$$

characterised by the odd numbers $k$, with $1<k<n-1$ modulo the involution $k \mapsto 2-k$.

Independently of $k$, their minimal field of definition $K$ is the splitting subfield of the prime $p$ in $\mathbf{Q}\left(\zeta_{n-1}\right)$.

Proof. Clearly, $a_{0}^{i}$ and $b_{0}^{j}$ generate the same automorphism group $A$ as $a_{0}$ and $b_{0}$, and by Lemma 4 they correspond to a regular dessin of the same type if their product has the same order $(n-1) / 2$ as $a_{0} b_{0}$. The additive constant is irrelevant: renumbering the elements of $\mathbf{F}_{n}$ by a translation makes it disappear. In the same way one may check that replacing $a_{0}$ and $b_{0}$ with $a_{0}^{i}$ and $b_{0}^{j}$, respectively, amounts to replacing $(u, k)$ with $\left(u^{s}, k^{\prime}\right)$. Then, as an element of $\mathrm{AGL}_{1}\left(\mathbf{F}_{n}\right)$, the action is given by

$$
a_{0}^{i} b_{0}^{j}: t \longmapsto(-1)^{i+j} u^{-i k+j k-2 j} t+c
$$

for some constant $c$, where we can omit the factor $(-1)$ since $i$ and $j$ are assumed to be odd. Thus the choice of $S$ guarantees the correct order, and the claim follows directly from Theorem 3 with some rather obvious calculations. The determination of $K$ relies on the same argument as in the proof of Theorem 4 .

ExAmPle 10. Using the involution $k \mapsto 2-k$, we can restrict attention to odd $k$ with $1<k \leqslant(n-1) / 2$.

755 (a) The first case to consider is $p=n=7$, leading with $k=3$ to two isomorphism classes of regular hypermaps of type $(7,3,3)$ and genus 3 , both on Klein's quartic; compare with $[\mathbf{2 3}]$ or Example 4.

(b) The next case is $p=n=11$, leading with $k=3$ and 5 to two Galois orbits, each containing four regular dessins of types $(5,5,5)$ and $(11,5,5)$ in genera 12 and 15 , all defined over $\mathbf{Q}\left(\zeta_{10}\right)=\mathbf{Q}\left(\zeta_{5}\right)$ (where the prime 11 splits completely). These orbits are special cases of Examples 5 and 4 .

(c) The first really new case occurs for $n=27, p=3$, and $e=3$, giving five Galois orbits of regular dessins of type $(13,13,13)$ for $k=3,5,7,9,11$ and one Galois orbit of type $(3,13,13)$ for $k=13$, all defined over the biquadratic field $K=\mathbf{Q}\left(\zeta+\zeta^{3}+\zeta^{9}\right)$, with $\zeta=\zeta_{26}$. 
As always, it is much more demanding to find explicit equations for the curves than to determine the fields of definition. We restrict our consideration to an accessible but already sufficiently complicated subcase of the regular hypermaps considered in Theorem 5 . In the notation and under the hypothesis given there, we concentrate on the case

$$
k:=\frac{n-1}{2}, \quad m_{0}=p, \quad m_{1}=\frac{n-1}{2(n-1, k-2)}=\frac{n-1}{2}
$$

(recall that $n \equiv 3 \bmod 4$, and hence $k$ and $k-2$ are odd) and the subfamily

$$
\begin{aligned}
\left\{H_{r} \mathcal{M}\left(n, u, \frac{n-1}{2}\right)^{*}, r \in(\mathbf{Z} /(n-1) \mathbf{Z})^{*}\right\} & =\left\{H_{r, r} \mathcal{M}\left(n, u, \frac{n-1}{2}\right)^{*}, r \in(\mathbf{Z} /(n-1) \mathbf{Z})^{*}\right\} \\
& =\left\{\mathcal{M}\left(n, u^{s}, \frac{n-1}{2}\right)^{*}, s \in(\mathbf{Z} /(n-1) \mathbf{Z})^{*}\right\} .
\end{aligned}
$$

According to Theorem 3, they form a Galois orbit, therefore it is sufficient to determine equations for $s=1$. Recall that $\mathbf{Q}\left(\zeta_{n-1}\right)=\mathbf{Q}\left(\zeta_{k}\right)$, and that the splitting subfield of the prime $p$ in $\mathbf{Q}\left(\zeta_{k}\right)$ is the fixed field of the subgroup of $\mathrm{Gal} \mathbf{Q}\left(\zeta_{k}\right) / \mathbf{Q}$ generated by $\zeta_{k} \mapsto \zeta_{k}^{p}$.

Theorem 6 . Let $n>3$ be a prime power $p^{e} \equiv 3 \bmod 4$. An affine (singular) model of the curve determined by the regular dessin $\mathcal{M}(n, u,(n-1) / 2)^{*}$ is given by e equations in $\mathbf{C}^{e+1}$ of type

$$
y_{\nu}^{p}=\prod_{j}\left(z-\zeta^{-p^{\nu} j}\right)^{\mu_{j}}, \quad \zeta:=\zeta_{k}, \quad k=\frac{n-1}{2}, \quad \nu=0, \ldots, e-1 .
$$

The integer exponents $\mu_{j}$ depend on the interplay between the additive and the multiplicative structures of $\mathbf{F}_{n}$ and can be calculated explicitly.

Proof. We consider a regular dessin of type $(p, k, k)$ and with an automorphism group $A \cong C_{p}^{e} \rtimes C_{k}$, defined as the index 2 subgroup of $\mathrm{AGL}_{1}\left(\mathbf{F}_{n}\right)=\left\{x \mapsto a x+b \mid a \in \mathbf{F}_{n}^{*}, b \in \mathbf{F}_{n}\right\}$ for which $a$ runs only over the cyclic group $C_{k}$ of squares in $\mathbf{F}_{n}^{*}$. The normal subgroup $\left(\mathbf{F}_{n},+\right)$ of translations is isomorphic to $C_{p}^{e}$, and we can fix three generators $g_{j}$ of $A$ acting on $\mathbf{F}_{n}$ as

$$
g_{0}: x \longmapsto x+1, \quad g_{1}: x \longmapsto u^{-2} x, \quad g_{\infty}: x \longmapsto u^{2}(x-1),
$$

where $u$ denotes, as before, a generator of the multiplicative group of the field. The respective orders of the generators are $p, k$ and $k$, and they satisfy $g_{0} g_{1} g_{\infty}=1$. The kernel $N$ of the homomorphism $h$ of $\Delta=\langle p, k, k\rangle$ onto $A$ given by

$$
\gamma_{j} \longmapsto g_{j}, \quad j=0,1, \infty
$$

is the surface group of the curve $X$. First we calculate the signature of the normal subgroup $\Gamma_{1}:=h^{-1}\left(C_{p}^{e}\right)$ of $\Delta$. To do so, we determine the cycle structure of the action of each generator on the cosets of $C_{p}^{e}$. Considered as permutations, they act as

$$
g_{0} \longmapsto(1), \quad g_{1} \longmapsto(12 \ldots k), \quad g_{\infty} \longmapsto(k \ldots 21) .
$$

Using Singerman's procedure described in [20] and the Riemann-Hurwitz formula, we can deduce from this information that $\Gamma_{1}$ has genus 0 and signature $\langle 0 ; p, p, \ldots, p\rangle$ with $k$ generators $\gamma_{0}, \gamma_{1}^{-1} \gamma_{0} \gamma_{1}, \ldots, \gamma_{1}^{-(k-1)} \gamma_{0} \gamma_{1}^{k-1}$ of order $p$, mapped by $h$ to the elements as follows:

$$
g_{0}: x \longrightarrow x+1, \quad g_{1}^{-1} g_{0} g_{1}: x \longmapsto x+u^{2}, \ldots, \quad g_{1}^{-(k-1)} g_{0} g_{1}^{k-1}: x \longmapsto x+u^{n-3} .
$$

Therefore we can consider the covering $\beta: \Gamma_{1} \backslash \mathbf{H} \rightarrow \Delta \backslash \mathbf{H}$ as a cyclic cover of the Riemann sphere over itself of order $k$ and ramified in two points. In the usual normalisation, $\beta$ ramifies over 1 and $\infty$. We renormalise $\beta$ defining $z:=1-\beta$ so that the covering looks like $z \mapsto z^{k}$, 
ramified in and over 0 and $\infty$, and so that the covering group is the group of $k$ th roots of unity acting by multiplication. The critical values of the covering $N \backslash \mathbf{H} \rightarrow \Gamma_{1} \backslash \mathbf{H}$ can be identified with these roots of unity $\zeta^{j}, j \bmod k$, and the function field of $X$ is clearly a Kummer extension of $\mathbf{C}(z)$, that is a composite extension of $e$ cyclic field extensions of degree $p$ corresponding to $e$ index $p$ subgroups $U$ of $C_{p}^{e}$.

We concentrate first on the more difficult case $e>1$, identify $C_{p}^{e}$ with an $e$-dimensional vector space over $\mathbf{F}_{p}$ and consider its subgroup $U$ as an $e-1$-dimensional subspace. Besides 0 it contains as many $\mathbf{F}_{n}$-squares as non-squares since $U \backslash\{0\}$ splits into disjoint orbits under the multiplicative action of $\mathbf{F}_{p}^{*}$, and all these orbits consist of $\frac{p-1}{2}$ squares and $\frac{p-1}{2}$ non-squares: the non-squares. Thus the action of $g_{1}^{-j} g_{0} g_{1}^{j}: x \mapsto x+u^{2 j}$ on the cosets of $U$ is either trivial if $u^{2 j} \in U$ (in $\left(p^{e-1}-1\right) / 2$ cases) or given by a cycle of order $p$ if $u^{2 j} \notin U$.

In the trivial case the generator $\gamma_{1}^{-j} \gamma_{0} \gamma_{1}^{j}$ of $\Gamma_{1}$ also belongs to the index $p$ subgroup $\Gamma_{U}:=$ $h^{-1}(U)$, and therefore the cyclic covering $\Gamma_{U} \backslash \mathbf{H} \rightarrow \Gamma_{1} \backslash \mathbf{H}$ is unramified over the fixed point of this generator. If we suppose that $\gamma_{1}$ acts on the Riemann sphere $\Gamma_{1} \backslash \mathbf{H}$ as $z \mapsto \zeta z$, and that $z=1$ is the fixed point of $\gamma_{0}$ under its action, then $\zeta^{-j}$ is the fixed point of the generator $\gamma_{1}^{-j} \gamma_{0} \gamma_{1}^{j}$. The function field of $\Gamma_{U} \backslash \mathbf{H}$ is therefore $\mathbf{C}(z, y)$ with

$$
y^{p}=\prod_{u^{2 j} \notin U}\left(z-\zeta^{-j}\right)^{\mu_{j}}
$$

for some integer exponents $\mu_{j}$ to be determined as follows. In the non-trivial case the covering is ramified of order $p$ over this point $\zeta^{-j}$. Since the local behaviour of $\gamma_{1}^{-j} \gamma_{0} \gamma_{1}^{j}$ around its fixed point induces a multiplication of $y$ with $\zeta_{p}$, and since on the other hand the different branches of $y$ correspond to the different cosets of $U$, we can determine the $\mu_{j}$ in the following way. For simplicity suppose that $1=u^{0} \notin U$ such that $\gamma_{0} \notin \Gamma_{U}$ and such that the covering ramifies over $z=1$. We label the residue classes of $U$ in $C_{p}^{e} \cong \mathbf{F}_{n}$ (corresponding to the branches of $y$ ) such that

$$
U_{\mu}:=U+\mu \quad \text { for } \quad \mu=0,1, \ldots, p-1
$$

and such that $\gamma_{0}$ acts on the branches, here on the indices of the residue classes $U_{\mu}$, as the cyclic permutation $\tau=(01, \ldots, p-1)$. Clearly, if $\gamma_{1}^{-j} \gamma_{0} \gamma_{1}^{j} \notin U$, then it acts as the power $\tau^{\mu_{j}}$, where $\mu_{j}$ is determined by

$$
U+u^{2 j}=U_{\mu_{j}}=U+\mu_{j}
$$

(in particular $\mu_{0}=1$ ). With this normalisation for the other $\mu_{j}$ the first equation is determined. The explicit calculation of the $\mu_{j}$ depends on the choice of $U$ and requires a non-trivial comparison between the additive and the multiplicative structures of $\mathbf{F}_{n}$.

We can apply this procedure for any index $p$ subgroup of $C_{p}^{e} \cong \mathbf{F}_{n}$. On the other hand, it is clear that $e$ of these subgroups are sufficient provided their common intersection is 0 . This is equivalent to the property that their one-dimensional duals $\hat{U} \subset \hat{\mathbf{F}}_{n} \cong \mathbf{F}_{n}$ generate $\mathbf{F}_{n}$ as an additive group. By the normal basis theorem we can choose some Galois orbit as a basis for the field extension $\mathbf{F}_{n} / \mathbf{F}_{p}$. By dualisation, this means that we can choose a subspace $U$ with the property that by taking $p^{\nu}$-powers, with $\nu=0, \ldots, e-1$, we obtain $e$ subspaces $U^{p^{\nu}}$ with common intersection 0 . Their cosets are obtained by applying the Frobenius automorphisms to the cosets $U_{\mu}=U+\mu$ of $U$, of course. In the resulting equations the same effect is obtained if we replace $j$ with $p^{\nu} j$ : observe that $u^{2 j} \in(U+\mu)^{p^{e-\nu}}$ is equivalent to $u^{2 j p^{\nu}} \in(U+\mu)^{p^{e}}=U+\mu$.

The case where $e=1$ and $n=p$ is considerably simpler since we have only to consider $U=\{0\}$, and the $u^{2 j}$ automatically have integer representatives. We can simply replace the definition (4) with $\mu_{j}:=u^{2 j}$, and then all other arguments, as far as needed, remain valid. 
REMARK 7. The construction shows again that the Galois automorphisms of $\mathbf{Q}(\zeta) / \mathbf{Q}$ leaving invariant the splitting field of $p$ induce permutations of the coordinates $y_{\nu}$ only, therefore inducing isomorphisms, as predicted by Theorem 5 .

ExAmple 11. We take up Example 10(c) with the dessins of type $(3,13,13)$. To determine the three equations explicitly, we have to make a good choice of the index 3 subspace $U$ in $\mathbf{F}_{27}$. For some generator $u$ of the multiplicative group $\mathbf{F}_{27}^{*}$, a GAP calculation [24] gives the exponents in $U \backslash\{0\}$ as follows:

$$
E(U)=\{3,16,4,17,6,19,12,25\} \subset \mathbf{Z} / 26 \mathbf{Z} .
$$

(Since $u^{13}=-1$, it would be sufficient to give the residue classes $3,4,6,12 \bmod 13$ that form in fact a difference set mod 13, see the remark below.) The images under the operation of $\mathrm{Gal} \mathbf{F}_{n} / \mathbf{F}_{p}$ correspond to the exponent sets $E(U), 3 E(U)$ and $9 E(U)$ having an empty intersection; thus we have $\bigcap U^{p^{\nu}}=\{0\}$, and hence a good choice of $U$. The $u$-exponents for the additive residue classes of $U$ can be calculated as follows:

$$
\begin{aligned}
& E\left(U_{1}\right)=\{0,1,2,8,11,18,20,22,23\}, \\
& E\left(U_{2}\right)=\{5,7,9,10,13,14,15,21,24\} .
\end{aligned}
$$

Therefore, the first of the three equations can be written as

$$
y_{0}^{3}=\prod_{2 j \in E\left(U_{1}\right)}\left(z-\zeta^{-j}\right) \prod_{2 j \in E\left(U_{2}\right)}\left(z-\zeta^{-j}\right)^{2},
$$

the other two for $y_{1}$ and $y_{2}$ following from this by replacing $\zeta^{-j}$ with $\zeta^{-3 j}$ and $\zeta^{-9 j}$, respectively.

REMARK 8. The prime number cases $n=p$ can be treated as in [22]; see Example 4; there, we take $q=k, s=-1$ and the replace $u$ with $u^{2}$. In the next case $n=p^{3}$, we may consider the two-dimensional subspaces $U \subset \mathbf{F}_{p^{3}}$ as lines in the (cyclic) projective plane $\mathbf{P}^{2}\left(\mathbf{F}_{p}\right)$, therefore it is not surprising that its elements not equal to 0 , seen as exponents of a fixed generator $u$ of $\mathbf{F}_{p^{3}}$, correspond to the elements of a difference set $\bmod \left(p^{3}-1\right) /(p-1)$. A similar rule is true for $p$-exponents $e>3$; for example, for $e=5$ we get the elements of $U$ via generalised difference sets corresponding to the points of a projective 3 -space in $\mathbf{P}^{4}\left(\mathbf{F}_{p}\right)$.

\section{Appendix}

A regular map on an orientable surface is said to be reflexible if it has an orientation-reversing automorphism.

Lemma A.1. Let $\mathcal{M}$ be a reflexible embedding of a graph $\mathcal{G}$ of girth 3 and prime valency $p$ such that $(p-1) / 2$ is also prime (and thus a Sophie Germain prime). Then Aut $\mathcal{G}=$ Aut $\mathcal{M}, \quad{ }^{875}$ the full automorphism group of $\mathcal{M}$, including orientation-reversing elements.

Proof. Clearly Aut $\mathcal{G} \geqslant$ Aut $\mathcal{M}$. Since $\mathcal{M}$ is reflexible, it follows that the subgroup (Aut $\mathcal{M})_{v}$ of Aut $\mathcal{M}$ fixing a vertex $v$ acts on the set $N(v)$ of neighbouring vertices of $v$ as the dihedral group $D_{p}$, and thus the subgroup $(\text { Aut } \mathcal{G})_{v}$ of Aut $\mathcal{G}$ fixing $v$ induces on $N(v)$ a permutation group $P$ of degree $p$ containing $D_{p}$. By theorems of Galois and Burnside, any transitive group of prime degree $p$ is either doubly transitive or is a proper subgroup of the affine group $\operatorname{AGL}_{1}\left(\mathbf{F}_{p}\right)$.

First suppose that $P$ is doubly transitive. Since $\mathcal{G}$ has girth 3 , it contains a triangle, and since Aut $\mathcal{G}$ acts transitively on the vertices of $\mathcal{G}$, we may assume that $v$ is a vertex of this triangle. 
Thus at least two of the neighbours of $v$ are adjacent, and since (Aut $\mathcal{G})_{v}$ is doubly transitive on $N(v)$, they are all adjacent. Thus $v$ and $N(v)$ span a complete subgraph $K(v) \cong K_{p+1}$ in $\mathcal{G}$, and since $\mathcal{G}$ is connected and has valency $p$, it follows that $\mathcal{G}=K(v)$. However, the regular embeddings of complete graphs $K_{n}$ are all known [12], and for $n>4$ none of them is reflexible; this contradiction therefore shows that $P$ is a proper subgroup of $\operatorname{AGL}_{1}\left(\mathbf{F}_{p}\right)$. Now $P$ contains $D_{p}$, which is a maximal subgroup of $\operatorname{AGL}_{1}\left(\mathbf{F}_{p}\right)$ since its index $(p-1) / 2$ is prime, and hence $P=D_{p}$.

The stabiliser in $D_{p}$ of any two points is the identity subgroup, and hence the kernel $K(v)$ of the action of $(\text { Aut } \mathcal{G})_{v}$ on $N(v)$ consists of those $g \in$ Aut $\mathcal{G}_{v}$ fixing at least two neighbours $w$ of $v$. Since $\mathcal{G}$ contains a triangle and $\mathcal{M}$ is regular, such a pair $v, w$ have a common neighbour $u$ in $\mathcal{G}$, so $g$ fixes at least two elements $u, v$ of $N(w)$, so $g \in K(w)$. Thus $K(v) \leqslant K(w)$, so the connectedness of $\mathcal{G}$ implies that $K(v)=K\left(v^{\prime}\right)$ for all vertices $v, v^{\prime}$ of $\mathcal{G}$. Since $\mathcal{G}$ has no multiple edges, it follows that $K(v)=1$, and hence $(\text { Aut } \mathcal{G})_{v} \cong D_{p}$. Thus $(\text { Aut } \mathcal{G})_{v}=(\text { Aut } \mathcal{M})_{v}$ and hence Aut $\mathcal{G}=\operatorname{Aut} \mathcal{M}$.

Note that one cannot remove the condition that $\mathcal{G}$ has girth 3: for instance, the Fermat curve of degree $p$ gives a reflexible embedding $\mathcal{M}$ of the complete bipartite graph $\mathcal{G}=K_{p, p}$, which has girth 4 , with Aut $\mathcal{M} \cong D_{p} \times D_{p}$ of order $4 p^{2}$ and Aut $\mathcal{G}$ isomorphic to the wreath product $S_{p} \prec S_{2}$ of order $2(p !)^{2}$. Similarly, the reflexibility condition is essential here: for each Mersenne prime $p$ the complete graph $\mathcal{G}=K_{p+1}$, of girth 3 and valency $p$, has chiral (that is regular but not reflexible) embeddings $\mathcal{M}$ with Aut $\mathcal{M} \cong \operatorname{AGL}_{1}\left(\mathbf{F}_{p+1}\right)$ and Aut $\mathcal{G} \cong S_{p+1}$; see [12].

We can apply Lemma A.1 to the Macbeath-Hurwitz dessins, which have valency 7 and girth

3 , and are reflexible since the corresponding curves are defined over real fields.

Corollary A.1. Let $p$ be a prime congruent to $\pm 1 \bmod 7$. Then the graphs underlying the three Galois conjugate Macbeath-Hurwitz dessins of type $(7,2,3)$ and automorphism group $\mathrm{PSL}_{2}\left(\mathbf{F}_{p}\right)$ are pairwise non-isomorphic.

Proof. According to Lemma A.1, any isomorphism between two of them would extend to

\section{References}

1. P. Beazley Cohen, C. Itzykson and J. Wolfart, 'Fuchsian triangle groups and Grothendieck dessins: variations on a theme of Belyi', Comm. Math. Phys. 163 (1994) 605-627.

2. G. V. Bely I, 'On Galois extensions of a maximal cyclotomic field', Izv. Akad. Nauk SSSR Ser. Mat. 43 (1979) 267-276, 479.

3. N. L. Biggs, 'Automorphisms of imbedded graphs', J. Combin. Theory Ser. B 11 (1971) 132-138.

4. K. Coombes and D. Harbater, 'Hurwitz families and arithmetic Galois groups', Duke Math. J. 52 (1985) $821-839$.

5. R. CORI, 'Un code pour les graphes planaires et ses applications' Astérisque 27 (Société Mathématique de France, Paris, 1975).

6. A. Coste, G. A. Jones, M. Streit and J. Wolfart, 'Generalised Fermat hypermaps and Galois orbits', Glasg. Math. J. 51 (2009) 289-299.

7. H. S. M. Coxeter and W. O. J. Moser, Generators and relations for discrete groups (Springer, Berlin 1980).

8. P. Dèbes and M. Emsalem, 'On fields of moduli of curves', J. Algebra 211 (1999) 42-56.

9. A. Grothendieck, 'Esquisse d'un Programme', Geometric Galois actions 1. Around Grothendieck's Esquisse d'un Programme (ed. P. Lochak and L. Schneps) London Mathematical Society Lecture Note Series 242 (Cambridge University Press, Cambridge, 1997) 5-48.

10. B. Huppert, Endliche Gruppen I (Springer, Berlin, 1967).

930 11. L. D. JAMEs, 'Edge-symmetric orientable imbeddings of complete graphs', European J. Combin. 11 (1990) $133-144$.

12. L. D. James and G. A. Jones, 'Regular orientable imbeddings of complete graphs', J. Combin. Theory Ser. B 39 (1985) 353-367. 
13. G. A. Jones, R. Nedela and M. Škoviera, 'Regular embeddings of $K_{n, n}$ where $n$ is an odd prime power', European J. Combin. 28 (2007) 1863-1875.

14. G. A. Jones and D. Singerman, 'Belyi functions, hypermaps and Galois groups', Bull. London Math Soc. 28 (1996) 561-590.

15. G. A. Jones and M. Streit, 'Galois groups, monodromy groups and cartographic groups', Geometric Galois actions 2. The inverse Galois problem, moduli spaces and mapping class groups (ed. P. Lochak and L. Schneps) London Mathematical Society Lecture Note Series 243 (Cambridge University Press, Cambridge, 1997) 25-65.

16. G. A. Jones, M. Streit and J. Wolfart, 'Galois action on families of generalised Fermat curves', J. Algebra 307 (2007) 829-840.

17. G. A. Jones and D. B. Surowski, 'Regular cyclic coverings of the Platonic maps', European J. Combin. 21 (2000) 333-345.

18. G. A. Jones and J. S. Thornton, 'Operations on maps, and outer automorphisms', J. Combin. Theory Ser. B 35 (1984) 93-103.

19. A. M. Maсbeath, 'Generators of the linear fractional groups', Number theory, (ed. W. J. Leveque and E. G. Straus) Proceedings of Symposia in Pure Mathematics XII, Houston, TX, 1967 (American Mathematical Society, Providence, RI, 1969) 14-32.

20. D. Singerman, 'Finitely maximal Fuchsian groups', J. London Math. Soc. (2) 6 (1978) 29-38.

21. M. Streit, 'Field of definition and Galois orbits for the Macbeath-Hurwitz curves', Arch. Math. 74 (2000) 342-349.

22. M. Streit and J. Wolfart, 'Characters and Galois invariants of regular dessins', Rev. Mat. Complut. 13 (2000) 49-81.

23. M. Streit and J. Wolfart, 'Cyclic projective planes and Wada dessins', Doc. Math. 6 (2001) 39-68.

24. The GAP Group, 'GAP - groups, algorithms, and programming', http://www.gap-system.org.

25. V. A. Voevodsky and G. B. Shabat, 'Equilateral triangulations of Riemann surfaces and curves over algebraic number fields', Soviet Math. Dokl. 39 (1989) 38-41.

26. T. R. S. WAlsh, 'Hypermaps versus bipartite maps', J. Combin. Theory Ser. B 18 (1975) 155-163.

27. S. E. Wilson, 'Operators over regular maps', Pacific J. Math. 81 (1979) 559-568.

28. J. Wolfart, 'The 'obvious' part of Belyi's theorem and Riemann surfaces with many automorphisms', Geometric Galois actions 1 (ed. L. Schneps and P. Lochak) London Mathematical Society Lecture Note Series 242 (Cambridge University Press, Cambridge, 1997) 97-112.

29. J. Wolfart, 'ABC for polynomials, dessins d'enfants, and uniformization-a survey', Elementare und Analytische Zahlentheorie (Tagungsband), Proceedings ELAZ-Conference, 24-28 May, 2004 (ed. W. Schwarz and J. Steuding; Steiner, Stuttgart, 2006) http://www.math.uni-frankfurt.de/ wolfart/.

G. A. Jones

School of Mathematics

University of Southampton

Southampton

SO17 1BJ

United Kingdom

G.A.Jones@maths.soton.ac.uk

\section{J. Wolfart}

Mathematisches Seminar der Goethe Universität

Postfach 111932

D-60054 Frankfurt a.M.

Germany

wolfart@math.uni-frankfurt.de

\section{Streit}

Usinger Str. 56

D-61440 Oberursel

Germany

Manfred.Streit@bahn.de 